\title{
Galvanic Vestibular Stimulation: Cellular Substrates and Response Patterns of Neurons in the Vestibulo-Ocular Network
}

\author{
Kathrin D. Gensberger, ${ }^{1 *}{ }^{(0}$ Anna-Kristin Kaufmann, ${ }^{1 *}$ Haike Dietrich, ${ }^{1,2,3}$ Francisco Branoner, ${ }^{1}$ Roberto Banchi, ${ }^{1,2,3}$ \\ -Boris P. Chagnaud, ${ }^{1}$ and Hans Straka ${ }^{1,3}$ \\ ${ }^{1}$ Department Biology II, ${ }^{2}$ Graduate School of Systemic Neurosciences, and ${ }^{3}$ German Center for Vertigo and Balance Disorders, Ludwig-Maximilians- \\ University Munich, 82152 Planegg, Germany
}

Galvanic vestibular stimulation (GVS) uses modulated currents to evoke neuronal activity in vestibular endorgans in the absence of head motion. GVS is typically used for a characterization of vestibular pathologies; for studies on the vestibular influence of gaze, posture, and locomotion; and for deciphering the sensory-motor transformation underlying these behaviors. At variance with the widespread use of this method, basic aspects such as the activated cellular substrate at the sensory periphery or the comparability to motion-induced neuronal activity patterns are still disputed. Using semi-intact preparations of Xenopus laevis tadpoles, we determined the cellular substrate and the spatiotemporal specificity of GVS-evoked responses and compared sinusoidal GVS-induced activity patterns with motion-induced responses in all neuronal elements along the vestibulo-ocular pathway. As main result, we found that, despite the pharmacological block of glutamatergic hair cell transmission by combined bath-application of NMDA (7-chloro-kynurenic acid) and AMPA (CNQX) receptor blockers, GVS-induced afferent spike activity persisted. However, the amplitude modulation was reduced by $\sim 30 \%$, suggesting that both hair cells and vestibular afferent fibers are normally recruited by GVS. Systematic alterations of electrode placement with respect to bilateral semicircular canal pairs or alterations of the bipolar stimulus phase timing yielded unique activity patterns in extraocular motor nerves, compatible with a spatially and temporally specific activation of vestibulo-ocular reflexes in distinct planes. Despite the different GVS electrode placement in semi-intact X. laevis preparations and humans and the more global activation of vestibular endorgans by the latter approach, this method is suitable to imitate head/body motion in both circumstances.

Key words: extraocular motor; galvanic stimulation; hair cells; inner ear; vestibular; Xenopus laevis

Significance Statement

Galvanic vestibular stimulation is used frequently in clinical practice to test the functionality of the sense of balance. The outcome of the test that relies on the activation of eye movements by electrical stimulation of vestibular organs in the inner ear helps to dissociate vestibular impairments that cause vertigo and imbalance in patients. This study uses an amphibian model to investigate at the cellular level the underlying mechanism on which this method depends. The outcome of this translational research unequivocally revealed the cellular substrate at the vestibular sensory periphery that is activated by electrical currents, as well as the spatiotemporal specificity of the evoked eye movements, thus facilitating the interpretation of clinical test results.

\section{Introduction}

Ever since LeRoy (1755) produced visual sensations by passing current through the eye and Galvani (1791) used current to evoke

\footnotetext{
Received Nov. 25, 2015; revised July 12, 2016; accepted July 14, 2016.

Author contributions: H.S. designed research; K.D.G., A.-K.K., H.D., F.B., R.B., B.P.C., and H.S. performed research; K.D.G., A.-K.K., H.D., F.B., R.B., B.P.C., and H.S. analyzed data; K.D.G., A.-K.K., H.D., F.B., R.B., B.P.C., and H.S. wrote the paper.

The authors acknowledge the assistance of Alexander Knorr with the eye motion analysis.

This work was supported by the Collaborative Research Center 870 (CRC 870) and the Graduate Program (GRK 1373) of the German Science Foundation, the German Federal Ministry of Education and Research (Grants 01 E0 0901 and 01 GQ 1407), and the Graduate School of Systemic Neurosciences at Ludwig-Maximilians-University Munich.
}

contractions of frog muscles, electrical stimulation has been used in neuroscientific research to activate excitable cellular elements (Thompson et al., 2014). After the descriptions of balance problems and equilibrium disturbances when passing current through the human head (Purkinje, 1820) or applying currents to both

\footnotetext{
The authors declare no competing financial interests.

*K.D.G. and A.K.K. contributed equally to this work.

Correspondence should be addressed to Hans Straka, Department Biology II, Ludwig-Maximilians-Universität Munich, Grosshaderner Str. 2, 82152 Planegg, Germany. E-mail: straka@Imu.de.

DOl:10.1523/JNEUROSCI.4239-15.2016

Copyright $\odot 2016$ the authors $\quad 0270-6474 / 16 / 369097-14 \$ 15.00 / 0$
} 
ears (Hitzig, 1871), galvanic vestibular stimulation (GVS) became routine to stimulate vestibular sense organs (Bos and Jongkees, 1963; Curthoys, 2010). So far, GVS has been used to investigate the role of vestibular signals in gaze, posture, and locomotor control, as well as motion and spatial perception in human subjects under pathophysiological conditions and in clinical practice (Fitzpatrick and Day, 2004; Curthoys, 2010; St George and Fitzpatrick, 2011; Hsu et al., 2012; Ferrè et al., 2013; Grasso et al., 2013; Fitzpatrick and Watson, 2015). In addition, GVS has assisted in deciphering cellular aspects of vestibular signal processing in various animal models (Goldberg, 2000).

Galvanic currents influence the discharge of otolith and semicircular canal nerve afferents (Goldberg et al., 1984; Schneider et al., 2002; Fitzpatrick and Day, 2004; Kim and Curthoys, 2004; Curthoys and Macdougall, 2012); however, different approaches are used to achieve this goal. Stimulus electrodes in different vertebrate species were either inserted unilaterally or bilaterally into the perilymphatic space of the semicircular canals (Ezure et al., 1983; Goldberg et al., 1984; Angelaki and Perachio, 1993), placed in the middle ear cavity (Kim and Curthoys, 2004; Shanidze et al., 2012; Kim, 2013a, 2013b), or noninvasively attached to the neck for transmastoidal stimulation in human subjects (Fitzpatrick and Day, 2004). The latter two methods preserve inner ear function and thus permit experimental perturbations of motion-induced responses (Shanidze et al., 2012). Independent of the stimulus method, GVS at low intensity activates irregular vestibular afferents, whereas higher stimulus intensities also recruit regular afferents (Goldberg et al., 1984; Kim and Curthoys, 2004; Kim et al., 2011). This stimulus-amplitudedependent activation allows determining various details of vestibular signal processing, such as fiber-specific origins of monosynaptic and disynaptic inputs to central vestibular neurons (Highstein et al., 1987; Minor and Goldberg, 1991; Angelaki and Perachio, 1993; Straka and Dieringer, 2000) or the organization of vestibulo-motor and vestibulo-autonomous reflexes (Courjon et al., 1987; Cohen et al., 2011; Shanidze et al., 2012; Kim, 2013a).

However, despite an apparent consensus regarding induction and specificity of GVS-induced responses (Wardman and Fitzpatrick, 2002), no definitive experimental proof for the cellular substrate that is activated by GVS is yet available. Although some experimental studies assume a direct activation of vestibular afferents by GVS (Goldberg et al., 1984), recent clinical evidence indicates that GVS might also activate hair cells (Aw et al., 2013). Unequivocal knowledge about the activated structure, however, is necessary to differentiate between human patients suffering from Ménière's disease (Aw et al., 2013) or vestibular migraine (Clarke, 2010; Curthoys, 2010). To resolve basic functional principles of GVS, including activated substrates, studies on tractable animal models are required that allow stimulation of individual endorgans within intact inner ears, recordings from individual vestibulo-ocular reflex (VOR) neuronal elements, as well as pharmacological manipulations. Given the well described vestibuloocular signal processing in amphibians (Straka and Dieringer, 2004) and the highly conserved morpho-physiological organization of the VOR circuitry in vertebrates, including vestibular nerve afferent and extraocular motor activity patterns (Straka et al., 2014), Xenopus laevis tadpoles are an ideal model system with which to decipher basic aspects of GVS.

Here, we used semi-intact tadpole preparations to compare the induction and modulation of the activity in vestibular nerve afferents, central vestibular neurons, and extraocular motoneurons, as well as eye movements during head rotation and GVS.
Systematic manipulations of stimulus electrode position, stimulus parameters, and pharmacological block of the glutamatergic transmission were used to determine the spatiotemporal specificity and morphological substrate of GVS-evoked responses. Preliminary data were published in abstract form previously (Kaufmann et al., 2013).

\section{Materials and Methods}

Animals. X. laevis tadpoles of either sex were obtained from the in-house animal breeding facility at the Biocenter-Martinsried of the LudwigMaximilians-University Munich. Tadpoles were kept in tanks filled with $17-18^{\circ} \mathrm{C}$ nonchlorinated water at a $12 / 12$ light/dark cycle and were fed daily with Spirulina bacteria. A total of 88 animals at developmental stages 53-55 (Nieuwkoop and Faber, 1994) were used for this study. Experiments were performed in vitro on isolated, semi-intact preparations and comply with the National Institutes of Health publication entitled Principles of Animal Care No. 86-23 (revised 1985). Permission for these experiments was granted by the governmental institution at the Regierung von Oberbayern/Government of Upper Bavaria (55.2-1-54-2532.3-59-12).

Isolated semi-intact in vitro preparations. For all experiments, tadpoles were anesthetized in $0.05 \%$ 3-aminobenzoic acid ethyl ester (MS-222; Pharmaq) in frog Ringer's solution containing the following (in mM): 75 $\mathrm{NaCl}, 25 \mathrm{NaHCO}_{3}, 2 \mathrm{CaCl}_{2}, 2 \mathrm{KCl}, 0.5 \mathrm{MgCl}_{2}$, and 11 glucose, $\mathrm{pH} 7.4$, and decapitated at the level of spinal segments $5-10$. The skin covering the top of the head was removed, the soft skull tissue and rostral vertebrae opened, and the forebrain disconnected. This surgical procedure anatomically preserved all vestibular endorgans within the otic capsule on both sides (Fig. 1 $A, B$ ), the CNS, the extraocular motor innervation, and eye muscles and allowed prolonged in vitro experimentation (Ramlochansingh et al., 2014), including an in vivo-like activation of the VOR during motion or electrical stimulation (Straka and Simmers, 2012).

Vestibular and extraocular motor nerve recordings. For recordings of vestibular afferent activity, the VIII ${ }^{\text {th }}$ nerve was transected at the entrance into the brainstem, leaving the peripheral portion along with the ganglion of Scarpa and the sensory innervation of all vestibular endorgans in the otic capsule intact. The brain was removed to facilitate the visibility and access to the cut surface of the peripheral portion of the VIII ${ }^{\text {th }}$ nerve for electrophysiological recordings. For recordings of extraocular motor nerve activity, the skin surrounding the eye was removed and the nerve branches innervating the lateral rectus (LR) or the superior oblique (SO) eye muscles were disconnected from their target muscles. Thereafter, preparations were rinsed in freshly oxygenated Ringer's solution $\left(95 \% \mathrm{O}_{2}, 5 \% \mathrm{CO}_{2}\right.$; Carbogen) and mechanically secured to the Sylgard-lined floor of the recording chamber (volume, $5 \mathrm{ml}$ ). During the experiments, preparations were continuously superfused with oxygenated Ringer's solution at a rate of $\sim 2 \mathrm{ml} / \mathrm{min}$. The temperature of the bath solution was maintained at $17.0 \pm 0.2^{\circ} \mathrm{C}$.

Extracellular multiunit spike discharge from the isolated extraocular motor nerves (LR, SO; $n=44)$ or multiunit $(n=8)$ and single-unit activity of vestibular nerve afferent fibers $(n=39)$ was recorded by targeting glass suction electrodes with a micromanipulator to the cut end of the extraocular motor nerves or the fasciculated cut end of the VIII ${ }^{\text {th }}$ nerve. Glass microelectrodes were made with a horizontal puller (P-87; Sutter Instruments) and were individually adjusted at the tip to fit the diameter of the respective target nerves. Vestibular and extraocular motor nerve activity was recorded (EXT 10-2F; npi electronic), digitized at $10-20 \mathrm{kHz}$ (CED 1401, Cambridge Electronic Design) and stored on a computer for offline analysis. For the analysis, responses obtained during $8-60$ repetitions of sinusoidal turntable oscillations or sinusoidally modulated current stimuli were averaged to obtain the mean response over a single cycle. Data from particular sets of experiments were averaged and presented as mean \pm SEM or SD.

Eye motion recordings. After isolation, preparations were mechanically secured to the Sylgard floor of the recording chamber. Because of the maintained neuronal innervation of the extraocular muscles, this semiintact preparation allowed the activation of eye movements by vestibular stimulation (Straka and Simmers, 2012). As described previously (Ram- 
A
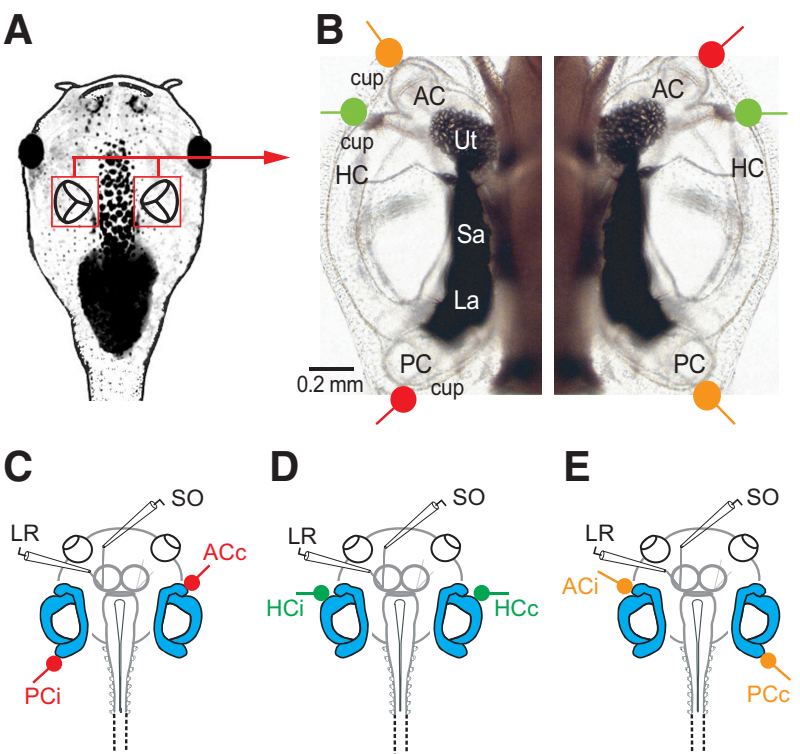

$\mathbf{F}$

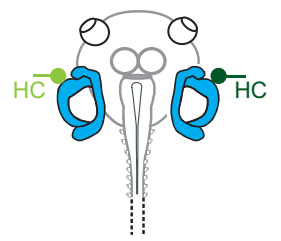

G

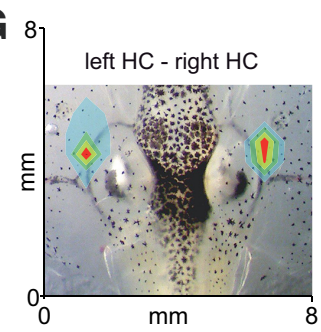

D

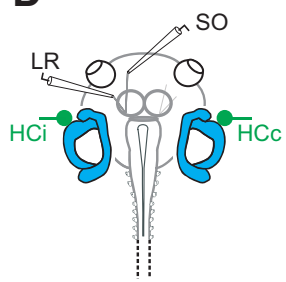

$\mathbf{E}$

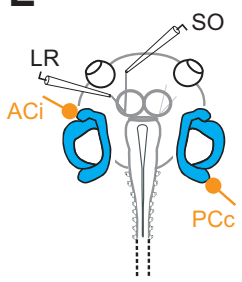

Galvanic current stimulus

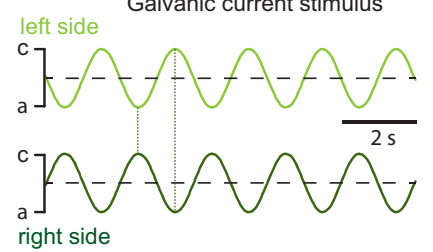

$\mathrm{H} 8$

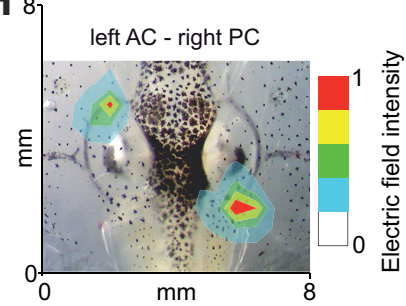

Figure 1. Anatomical substrate, electrode positions for extraocular motor nerve recordings, and application of galvanic vestibular current stimuli. $\boldsymbol{A}, \boldsymbol{B}$, Schematic of a semi-intact $X$. laevis preparation highlighting the location of the otic capsules $(\boldsymbol{A})$ and photomicrograph depicting the arrangement of bilateral vestibular endorgans and the location of the semicircular canal epithelia as marker for positioning the electrodes on the outer surface of the otic capsules for bipolar electrical stimulation of the three (red, green, orange) coplanar semicircular canal pairs, exemplarily shown in a stage 50 larva $(\boldsymbol{B})$. $\boldsymbol{C}-\boldsymbol{E}$, Schematics illustrating the location of the electrodes for $L R$ and $S 0$ motor nerve recordings, along with the three basic positions of the stimulus electrodes for activating plane-specific bilateral semicircular canal pairs. $\boldsymbol{F}-\boldsymbol{H}$, Sinusoidally modulated currents with alternating phase relation of the anodal/cathodal $(a, c)$ GVS currents $( \pm 100 \mu \mathrm{A})$ were applied through bilateral stimulus electrodes $(\boldsymbol{F})$; amplitude distribution plot of the generated electric field evoked by GVS of the bilateral $\mathrm{HCs}(\boldsymbol{G})$ and the left $\mathrm{AC}-$ right $\mathrm{PC}(\boldsymbol{H})$ superimposed on bright-field images of the respective head region; relative amplitude magnitudes are indicated by color code. Cup, Semicircular canal cupula; i,c, ipsilateral, contralateral with respect to the recorded extraocular motor nerve; La, lagena; Sa, saccule; Ut, utricle.

lochansingh et al., 2014), eye movements were recorded noninvasively with a video camera (Grasshopper color, Point Gray Research) and a zoom objective (Optem Zoom 70XL; Qioptiq Photonics) with an adequate lens $(\mathrm{M} 25 \times 0.75+0.25)$. This system was mounted on top of the experimental setup to visualize the motion of one eye from above during natural or galvanic vestibular stimulation at a video capture frame rate of $30 \mathrm{~Hz}$ with the FlyCap2 software (version 2.3.2.14). Eye motion profiles and parameters were extracted from the captured video sequences using a custom video-processing algorithm written in Matlab (for details, see Ramlochansingh et al., 2014). To calculate the motion of the eye, an ellipse was drawn around the eyeball and the angle between the minor

axis of the ellipse and the longitudinal axis of the head was calculated in each frame of a given video sequence (Beck et al., 2004). Based on the frame rate $(30 \mathrm{~Hz})$, the change in eye position over time was computed. The generation of peristimulus time histograms for a single cycle, obtained from 8-60 sinusoidal stimulus oscillations was used to calculate the eye motion gain (ratio eye/table motion).

Natural and galvanic vestibular stimulation. The recording chamber with the semi-intact $X$. laevis preparations was mounted on a computercontrolled, motorized two-axis turntable with the preparation centered in the horizontal and vertical rotation axes to provide optimal activation of semicircular canal organs (Lambert et al., 2008, 2013). Motion stimuli consisted of sinusoidal rotations across frequencies that ranged from 0.1 to $5.0 \mathrm{~Hz}$ (peak velocities: $\pm 6-60^{\circ}$ /s) in either the vertical (yaw) or horizontal (roll) axis to stimulate the bilateral horizontal or the ipsilateral posterior-contralateral anterior vertical semicircular canal pair preferentially as major modulatory vestibular inputs to LR and SO motoneurons, respectively. The limitation of motion stimulus frequencies to $5 \mathrm{~Hz}$ maximally is mainly due to the relatively soft cartilage of the X. laevis skull that prevents a firm fixation of the preparation to the recording chamber as tightly as in mammalian species, in which the bony skull is usually secured with a head holder to a stereotactic frame. In addition, the absence of considerable tissue above the neural targets, as well as the fluid pressure on the electrode shank, induced by inertia-related liquid motion at the Ringer's surface, impaired in particular stable recordings of single vestibular afferent fibers at higher rotation frequencies.

Sinusoidally modulated galvanic currents were applied by stimulus electrodes that consisted of two Teflon-coated silver wires (diameter: $0.76 \mathrm{~mm}$; AG 25-T; Science Products) placed on the outer surface of the otic capsule (Fig. 1 $A, B$ ). The two stimulus electrodes were cut at the tip, chlorinated to minimize polarization, and separately attached to a micromanipulator to enable precise positioning under visual guidance. The placement of the electrodes for GVS of a particular semicircular canal was facilitated by the clear visibility of all labyrinthine endorgans and sensory structures inside the transparent cartilaginous otic capsule of the semiintact larval $X$. laevis preparation (Fig. $1 A, B$ ). For most experiments, electrodes were placed bilaterally in close proximity of the visible cupulae of a specific coplanar semicircular canal pair (Fig. $1 B-E$ ). For the recording of vestibular nerve afferents, electrodes were placed unilaterally with one electrode close to either the horizontal or the anterior vertical semicircular canal cupula and the second electrode at a distance of $\sim 15 \mathrm{~mm}$ from the first in the Ringer's solution of the recording chamber. For most of the experiments, sine waves for GVS were produced with a linear stimulus isolator (WPI A395; World Precision Instruments), triggered by the analog output from a waveform function generator (Digital VCG Model 113; Wavetek) or by the analog output from an analog/digital converter (CED 1401). For $\mathrm{Ca}^{2+}$-imaging experiments, GVS sine waves were produced by a stimulus generator with an integrated isolation unit (STG 4004; Multichannel Systems). In most experiments, the galvanic currents were applied to the two electrodes in phase opposition and consisted of sinusoidally modulated currents at frequencies of 0.05 $10 \mathrm{~Hz}$ and amplitudes of $\pm 10-350 \mu \mathrm{A}$. The applied sinusoidal current generated an alteration of the cathode and anode between the two electrodes, respectively (Fig. $1 F$ ), and a local electric field that decreased in magnitude with the distance from the electrode (Fig. 1G,H). In some experiments, the sinusoidally modulated currents, applied to a bilateral semicircular canal pair, were in phase. In this case, the current was applied through two independently controlled sets of stimulus electrodes, each with one electrode close to the semicircular canal cupula and the second one at a distance of $\sim 15 \mathrm{~mm}$ from the first in the Ringer's solution of the recording chamber.

Imaging of $\mathrm{Ca}^{2+}$ transients in central vestibular neurons. $\mathrm{Ca}^{2+}$ transients were recorded in identified vestibulo-ocular neurons, retrogradely labeled from the midbrain oculomotor nucleus with a calcium sensor (Calcium Green-1 dextran, 3000 MW; Thermo Fisher Scientific). For this purpose, tadpoles were anesthetized in $0.05 \%$ MS-222, transferred into oxygenated, ice-cold Ringer's solution, and decapitated. After opening the skull on the ventral side and disconnecting the forebrain, the Ringer's solution was temporarily removed and crystals of Calcium Green-1 dextran, melted to an injection needle, were inserted unilaterally 
into the oculomotor nucleus, identified by the exit of the III ${ }^{\text {rd }}$ cranial nerve as described previously (Straka et al., 2001). Thereafter, surplus tracer was removed with excess Ringer's solution and preparations were incubated for $24-48 \mathrm{~h}$ at $14^{\circ} \mathrm{C}$ in oxygenated Ringer's solution. Imaging of $\mathrm{Ca}^{2+}$ transients in these vestibulo-ocular neurons during GVS of specific bilateral semicircular canal pairs was performed by using a fixed-stage microscope (Axio Examiner Z1; Carl Zeiss) equipped with a $40 \times(1.0)$ water-immersion objective and connected to a CCD camera (Axiocam HSM; Carl Zeiss). The microscope and camera were driven by a control unit and a signal distribution box (Examiner Control and SVB1; Carl Zeiss). Timing of GVS-induced image acquisition at a rate of 10-20 frames/s and data storage of the latter were controlled with the Axiovision software SE64 (Carl Zeiss). Fluorescence signal acquisition started $2-3$ s before the onset of the first GVS sine wave to determine the background fluorescence level $\left(F_{0}\right)$. Images were analyzed offline using the "intensity versus time" algorithm in the MBF-ImageJ Java software package (http://rsb.info.nih.gov/ij/). The background fluorescence was subtracted and bleaching effects were corrected using a linear regression algorithm written in IgorPro (Wavemetrics). All data are presented as relative changes in fluorescence $(\mathrm{d} F / F)$.

Pharmacology. Motion- or GVS-induced responses were completely blocked by bath application of a mixture of $15 \mu \mathrm{M}$ CNQX (Tocris Bioscience) and $50 \mu \mathrm{M}$ 7-chloro-kynurenic acid (7-Cl-KYNA; Tocris Bioscience). The two substances block AMPA- and NMDAreceptor-mediated components of glutamatergic transmission, respectively. A drug-related reduction of the responses in these isolated preparations usually occurred after 3-5 min and reached steady state after 15 min (Biesdorf et al., 2008). The reversible block of AMPA- and NMDA-receptor-mediated components by combined application of CNQX and 7-Cl-KYNA allowed evaluation of the neural substrate of galvanic stimulation as well as the specificity of the stimulated structures.

\section{Results}

\section{Experimental setting for galvanic vestibular stimulation}

Oppositely oriented sinusoidally modulated currents were applied to two stimulus electrodes, placed at the outer surface of the transparent otic capsule close to the cupulae of a bilateral coplanar semicircular canal pair, respectively (color-coded electrodes in Fig. $1 C-E$ ). These sinusoidal currents caused reciprocal oscillations of the anodal and cathodal current peaks at the electrode tips close to the semicircular canal sensory epithelia (Fig. $1 F$ ). To estimate the spatial extent of current spread during GVS, voltage amplitudes evoked by GVS of $\pm 100 \mu \mathrm{A}$ were systematically recorded with a glass microelectrode (tip diameter: $\sim 10 \mu \mathrm{m}$; resistance: $\sim 1 \mu \Omega$ ) that was filled with $2 \mathrm{M}$ sodium chloride. Recordings were made along a grid of $8 \times 6 \mathrm{~mm}$ ( $1 \mathrm{~mm}$ spacing) that covered the spatial dimensions of the preparation $(n=5)$ between the two stimulus electrodes (Fig. 1G,H). For evaluating the spatial extent of voltage magnitude distributions, two electrodes were placed close to the cupulae of either the bilateral horizontal semicircular canals (HC, Fig. 1G) or the anterior (AC) on the left and the posterior semicircular canal (PC) on the right side (Fig. $1 \mathrm{H}$ ). As indicated by the color-coded $2 \mathrm{D}$ voltage distribution plot, the peak amplitude at the site of the stimulus elec- trode dropped to $\sim 20 \%$ at a distance of $\sim 1 \mathrm{~mm}$. Therefore, this stimulation method causes only minimal current spread to susceptible structures other than the two targeted semicircular canal cupulae. In particular, the relatively distant location of the utricle $(\sim 1.5 \mathrm{~mm})$, saccule $(\sim 2 \mathrm{~mm})$, and lagena $(\sim 2 \mathrm{~mm})$ with respect to the closest semicircular canal stimulus electrode (Fig. 1B) minimizes an undesired electrical activation of otolith organs, suggesting that evoked responses are largely semicircular canal specific.

\section{Motion- and GVS-induced eye movements}

Horizontal sinusoidal rotation at a stimulus frequency of $0.5 \mathrm{~Hz}$ (red dashed line in Fig. 2A) provoked oscillatory movements of both eyes in isolated semi-intact in vitro preparations of $X$. laevis tadpoles $(n=9)$. Video recordings revealed the timing and amplitude of the evoked eye movements (red trace in Fig. $2 B$ ) that were oppositely oriented with respect to the rotation direction (Fig. 2C). Responses were approximately in phase with table position ( $T_{\text {pos }}$; gray trace in Fig. $2 C$ ) and thus phase-lagged with respect to table velocity ( $T_{\text {vel }}$; blue trace in Fig. $2 C$ ). Rotationevoked eye movements increased in amplitude with increasing stimulus magnitudes (Fig. 2E) with a gain (ratio eye/table motion) of $0.28 \pm 0.02$ at $\pm 30^{\circ} / \mathrm{s}$ peak velocity $\left(0.5 \mathrm{~Hz}, \pm 10^{\circ}\right.$ leftright table excursion; $n=9$ ). These responses were thus very similar, both in gain and phase, to the VOR observed under in vivo conditions for larval $X$. laevis at the same developmental stage (Lambert et al., 2008; Straka and Simmers, 2012).

In the same preparations that had been subjected to rotational stimulation $(n=9)$, eye movements were also elicited by sinu- 
A
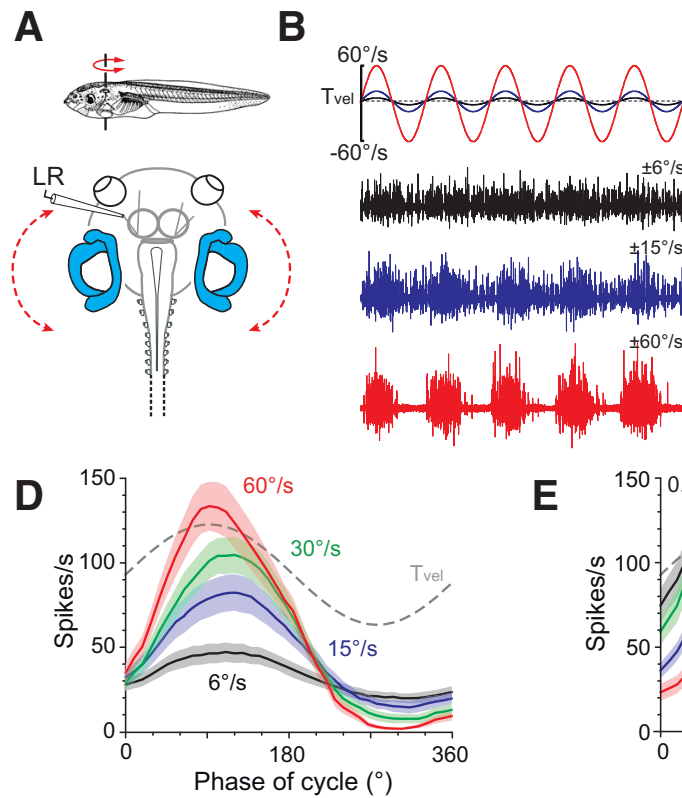

D

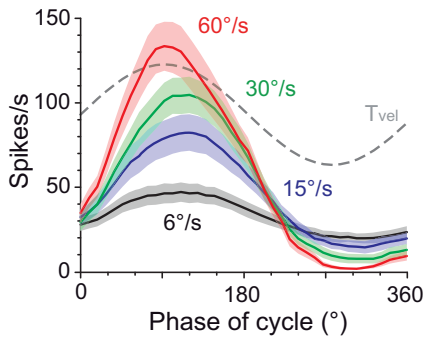

$\mathbf{F}$

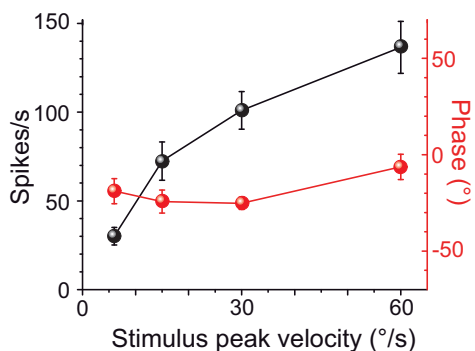

C
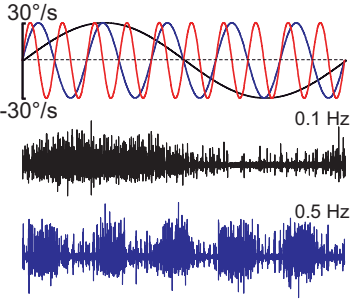$$
\text { - }
$$

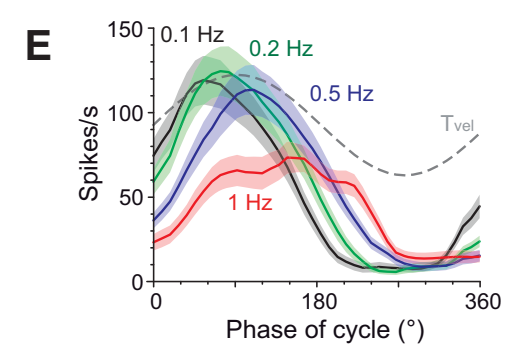

G

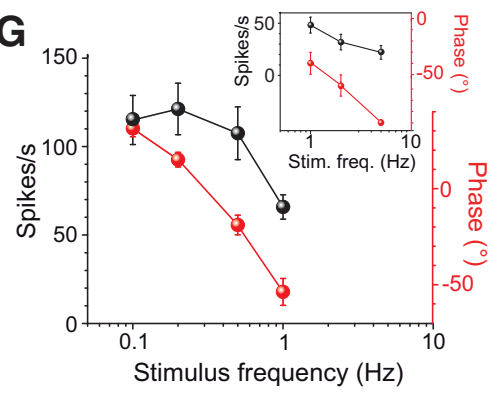

Figure 3. Response parameters of motion-induced extraocular motor activity. $\boldsymbol{A}$, Multiunit recordings of the $L R$ nerve during horizontal turntable rotation (red dashed arrow). $\boldsymbol{B}, \boldsymbol{C}, \mathrm{LR}$ nerve discharge during turntable rotation at a frequency of $0.5 \mathrm{~Hz}$ ( $\boldsymbol{B}$, top traces) and three peak velocities (black, blue, red trace); and a peak velocity of $\pm 30 \% \mathrm{~s}$ (C, top traces) and three stimulus frequencies (black, blue, red trace). $\boldsymbol{D}, \boldsymbol{E}$, Averaged firing rate modulation ( \pm SEM, shaded areas) over a single cycle (from 10 to 50 cycles, respectively; $n=10$ ) at $0.5 \mathrm{~Hz}$ and different peak velocities (color-coded traces in $\boldsymbol{D}$ ) and at $\pm 30 \%$ s and different stimulus frequencies (color-coded traces in $\boldsymbol{E}$ ); dashed lines in $\boldsymbol{D}$ and $\boldsymbol{E}$ indicate stimulus velocity. $\boldsymbol{F}, \boldsymbol{G}$, Dependency of response peak amplitude (black symbols in $\boldsymbol{F}, \boldsymbol{G}$ ) and phase (red symbols in $\boldsymbol{F}, \boldsymbol{G}$ ) of rotation-evoked cyclic LR nerve responses ( \pm SEM; $n=10$ ) with respect to stimulus peak velocity $(\boldsymbol{F})$ and frequency $(\boldsymbol{G})$. Inset in $\boldsymbol{G}$ shows data obtained in a separate set of experiments $(n=$ 7) in which the response dynamics at stimulus frequencies between 1 and $5 \mathrm{~Hz}$ were explored. Scale bar in $\boldsymbol{C}$ also applies to $\boldsymbol{B}$.

movements increased steadily until eventually saturation occurred at higher amplitudes. Direct comparison of current- and motion-evoked eye movements allowed calibrating GVS-induced eye movements with respect to eye motion, thus offering the possibility to use sinusoidal GVS to mimic a dynamically defined angular VOR behavior in the absence of effective motion. According to this calibration, imitation of a cyclic turntable rotation of, for example, $\pm 10^{\circ}$ leftright position excursion at $0.5 \mathrm{~Hz}$ (peak velocity: $\pm 30 \%$ s), required a GVS amplitude of approximately $\pm 180 \mu \mathrm{A}$ (black dashed lines in Fig. 2G).

\section{Motion-induced extraocular motor discharge}

To evaluate the general conditions for inducing eye movements with sinusoidal GVS, we first tested the impact of systematically altered motion stimulus parameters on the spatiotemporal specificity of extraocular motor activity. This evaluation was facilitated by the greater sensitivity to changes in stimulus conditions of extraocular motor spike discharge compared with effective eye motion. Therefore, the multiunit spike activity of disconnected and isolated extraocular motor nerves was recorded in semi-intact in vitro preparations (Straka and Simmers, 2012) during imposed head rotation that activated particular semicircular canal pairs (horizontal canals in Fig. $3 A$ ). Accordingly, during horizontal sinusoidal turntable rotation, the spontaneous multiunit discharge $(33.8 \pm 7.4$ spikes/s; $n=10)$ of the LR nerve was cyclically modulated (Fig. $3 B, C$ ).

With increasing peak velocities from soidal GVS of the bilateral HC cupulae at a stimulus frequency of $0.5 \mathrm{~Hz}$ [contralateral $\mathrm{HC}(\mathrm{HCc}) /$ ipsilateral $\mathrm{HC}(\mathrm{HCi})$ in Fig. 2A)]. GVS triggered horizontal oscillatory movements of both eyes with dynamic characteristics comparable to those observed during head rotation (cf. green and red traces in Fig. $2 B$ ). More specific, leftward movements of both eyes were generated during depolarization of the right $\mathrm{HC}$ ( $\mathrm{HCc}$; bottom gray trace in Fig. $2 B$ ) and concurrent hyperpolarization of the left HC (stimulus not plotted) and vice versa. This pattern complies with a reciprocal cathodal excitation and anodal inhibition of the two sensory epithelia, respectively, thus imitating rightward-leftward head oscillations. Temporal alignment of GVS-induced eye movements (green trace in Fig. 2D) and rotation-induced responses (red trace in Fig. 2C) indicated that the electrical stimulus reflected the velocity much more closely (blue arrowhead in Fig. $2 C)$ than the position component of the turntable motion (gray arrow in Fig. 2C). Therefore, with respect to evoked responses, GVS essentially imitates the velocity component of a head rotation.

With increasing head motion magnitude (Fig. 2E) as well as current intensity (Fig. $2 F$ ), the amplitude of the evoked eye $\pm 6 \%$ s to $\pm 60 \%$ s at a stimulus frequency of $0.5 \mathrm{~Hz}$ (Fig. $3 \mathrm{~B}$ ), the LR nerve peak discharge became larger, as indicated by the averaged responses over a single motion cycle, respectively, and reached up to $\sim 140$ spikes/s $(141.1 \pm 16.1$ spikes/s; $n=10)$ at a peak velocity of $\pm 60 \%$ (Fig. $3 D, F$ ). Whereas the spike rate increased steadily with peak velocity (black symbols in Fig. $3 F$ ), the corresponding phase lag of the responses of $\sim 10-20^{\circ}$ relative to stimulus velocity was largely independent of stimulus magnitude (red symbols in Fig. $3 F$ ). The overall change in LR nerve response amplitude and phase relation with increasing stimulus amplitude (Fig. $3 F$ ) is qualitatively very similar to that of rotation-induced eye movements (Fig. 2E). However, a direct translation of the presynaptic extraocular motor discharge into effective eye movements suffers from several unknown parameters, such as the dynamics at the neuromuscular junction and the required buildup and relaxation of muscle strength.

With increasing stimulus frequencies from 0.1 to $1 \mathrm{~Hz}$ at a peak velocity of $\pm 30 \%$ (Fig. $3 C$ ), the peak firing rate of the modulated responses decreased after reaching a maximum at $0.2 \mathrm{~Hz}$ (Fig. $3 E, G$ ). This decline (black symbols in Fig. $3 G$ ) was accompanied by a considerable change in the timing of the response 
peak over the range of applied stimuli (red symbols in Fig. 3G). In fact, responses evoked at a stimulus frequency of $0.1 \mathrm{~Hz}$ were phase advanced by $\sim 30^{\circ}\left(31.5^{\circ} \pm\right.$ $\left.4.3^{\circ} ; n=10\right)$, whereas those evoked at 1 $\mathrm{Hz}$ were phase lagged by $\sim 50^{\circ}\left(-49.8^{\circ} \pm\right.$ $\left.6.6^{\circ} ; n=17\right)$. In several preparations $(n=$ 7 ), successful LR nerve recordings were made during horizontal rotations at frequencies from 1 to $5 \mathrm{~Hz}$ (inset in Fig. $3 G$ ). With increasing stimulus frequencies $>1$ $\mathrm{Hz}$, the modulated peak firing rate decreased further to a peak firing rate of $\sim 20$ spikes $/ \mathrm{s}$ at $5 \mathrm{~Hz}(22.1 \pm 6.5 \mathrm{spikes} / \mathrm{s} ; n=$ $7)$, whereas the corresponding phase lagged stimulus velocity by $\sim 90^{\circ}\left(-92.6^{\circ}\right.$ $\pm 2.6^{\circ} ; n=7$; inset in Fig. $\left.3 G\right)$. These motion-induced extraocular motor responses and their respective dynamic characteristics were used in the following for a comparison with GVS-induced responses.

\section{GVS-induced extraocular motor discharge: response dynamics}

As a next step in evaluating the efficacy of GVS, we determined the correlation between extraocular motor discharge and stimulus current amplitude. Sinusoidal GVS of the bilateral HCs (Fig. 4A, top traces in $B$ ) caused a cyclic modulation of the multiunit LR nerve discharge $(n=8)$ that depended on the intensity of the applied current stimulus (color coded traces in Fig. 4B). The spontaneous LR nerve discharge of $\sim 35$ spikes/s was already noticeably modulated at a minimal current of $\pm 10 \mu \mathrm{A}$, as indicated by the averaged responses over a single cycle (black curve in Fig. 4E). With larger current amplitudes, the peak firing rate of the modulated multiunit spike activity gradually increased and reached $\sim 200$ spikes/s at an intensity of $\pm 100 \mu \mathrm{A}$ (Fig. $4 E$ ). Even larger current amplitudes of up to \pm 300 $\mu \mathrm{A}$, however, failed to further increase the peak firing rate, causing instead a saturation of the maximal discharge rate (green symbols in Fig. 4G) that was accompanied by a gradually increasing phase lead of the peak response (Fig. 4E). Comparable to the temporal characteristics of motionvelocity-induced responses of LR motoneurons (Fig. 3), the peak firing rate of the modulated LR nerve response approximately coincided with the peak of the GVS half cycle that simultaneously depolarized the HCc and hyperpolarized the $\mathrm{HCi}$ (Fig. 4E).

To generalize the findings obtained for the horizontal angular VOR, the evaluation of GVS-induced extraocular motor discharge modulation was extended onto other combinations of semicircular canals and extraocular motor nerves. Therefore, we recorded multiunit spike activity of the $\mathrm{SO}$ motor nerve during sinusoidal GVS of the ipsilateral posterior (PCi) and contralateral
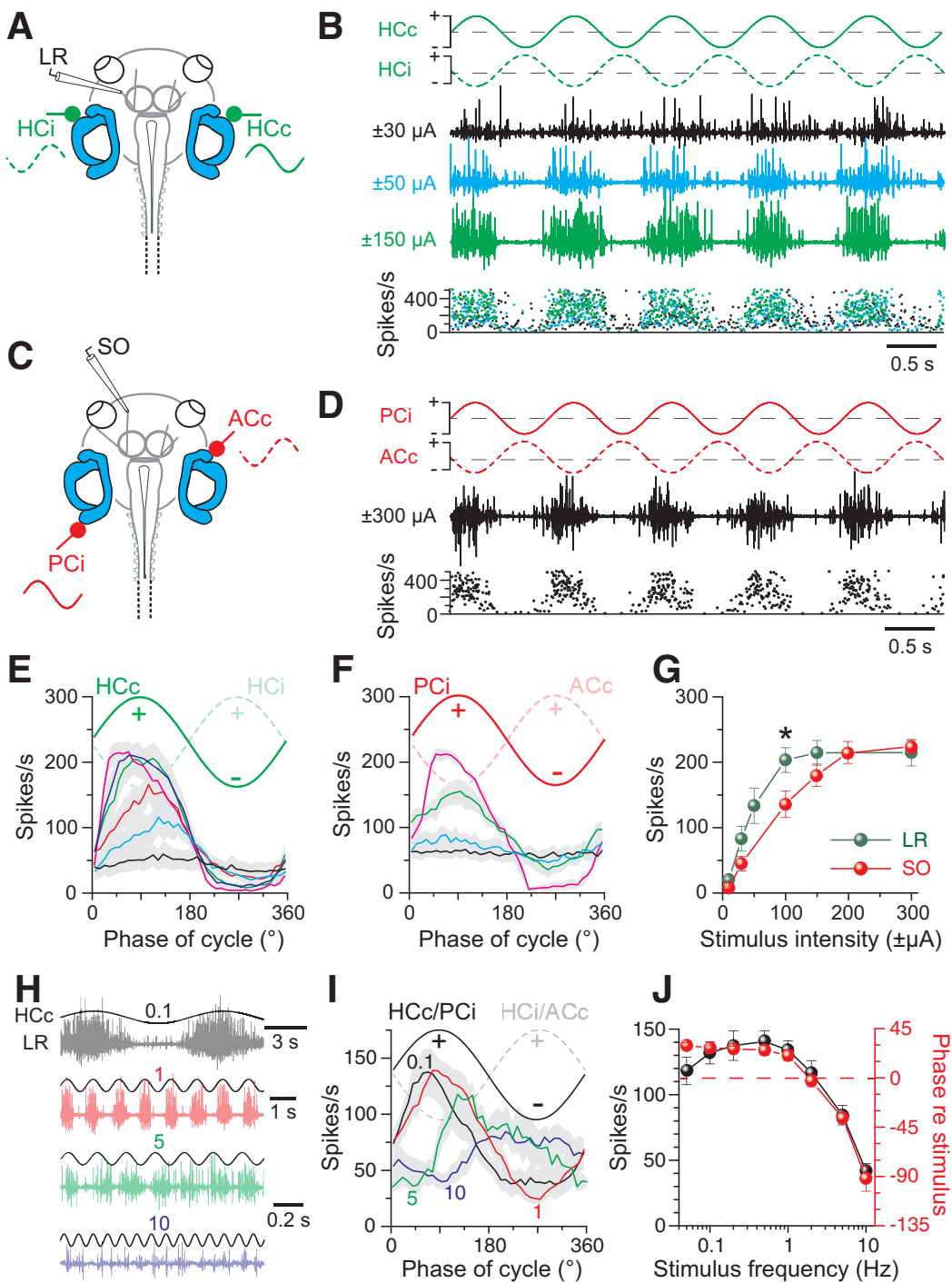

Figure 4. Frequency and intensity dependence of GVS-induced multiple-unit discharge modulation in extraocular motor nerves. $A, C$, Extracellular recordings of the left $L R(A)$ and SO $(\boldsymbol{C})$ nerves during GVS of the bilateral $\mathrm{HC}$ cupulae (green $\mathrm{HC}$ c and $\mathrm{HC}$ ) and the left $\mathrm{PC}(\mathrm{PCi})$ and right $\mathrm{AC}(\mathrm{ACC})$ cupulae, respectively. $\boldsymbol{B}$, Left LR nerve discharge during $1 \mathrm{~Hz}$ sinusoidal GVS of the bilateral $\mathrm{HC}$ cupulae (traces in top row) at three current intensities (black, blue, green traces) with peak firing rates (instantaneous rate, bottom plot) that increased with GVS amplitude. $\boldsymbol{D}$, Left SO nerve discharge (black trace) and instantaneous firing rate (bottom plot) during sinusoidal GVS of the left PC and right AC cupulae (traces in top row). $\boldsymbol{E}, \boldsymbol{F}$, Averaged $\mathrm{LR}(\boldsymbol{E}, n=8)$ and $\mathrm{S} 0$ nerve responses $(\boldsymbol{F}, n=8$ ) over a single GVS cycle at $1 \mathrm{~Hz}$ (from 16 cycles, \pm SEM, gray shaded areas) increased with stimulus amplitude (color-coded responses were evoked by increasing currents: $\pm 10, \pm 30, \pm 50, \pm 100, \pm 150, \pm 300 \mu \mathrm{A}$ in $E ; \pm 10, \pm 30, \pm 100$, $\pm 200 \mu \mathrm{A} \mathrm{in} \boldsymbol{F}) . \mathbf{G}$, Dependency of averaged LR and $\mathrm{S} 0$ nerve peak firing rates on GVS intensity; significance of difference between responses of the two nerves is indicated. ${ }^{*} p<0.05$ (Mann-Whitney $U$ test). $\boldsymbol{H}-\boldsymbol{J}$, LR nerve discharge during sinusoidal GVS ( \pm 100 $\mu \mathrm{A}$ ) of the bilateral $\mathrm{HCs}$ at four different stimulus frequencies (color-coded traces; $\boldsymbol{H}$ ); averaged LR/SO nerve responses (from 16 cycles, \pm SEM, gray shaded areas) over a single GVS cycle at $0.1,1,5$, and $10 \mathrm{~Hz}$ (color-coded curves) and a stimulus intensity of $\pm 100 \mu \mathrm{A}(n=8 ; I)$ reveal amplitude (black symbols) and phase dependency (red symbols) of the responses on stimulus frequency $(\boldsymbol{J})$. The stimulus in $\boldsymbol{H}$ indicates polarization of the HCc; numbers in $\boldsymbol{H}$ and $\boldsymbol{I}$ indicate frequency in $\mathrm{Hz}$.

anterior vertical semicircular canals (ACc; Fig. 4C). This latter bilateral semicircular canal pair is spatially coaligned with the pulling direction of the $\mathrm{SO}$ muscle and thus forms the major modulatory drive for the respective extraocular motoneurons (Branoner and Straka, 2015). Sinusoidally alternating GVS of the PCi and ACc (red traces in Fig. 4D) provoked a cyclic modulation (black trace in Fig. $4 D$ ) of the spontaneous multiunit SO nerve activity $(62.5 \pm 7.2$ spikes $/ \mathrm{s} ; n=8)$. The peak discharge of the modulated responses coincided with the GVS half cycle, during which the PCi was depolarized and the ACc was simultaneously 
hyperpolarized (Fig. 4F). A first modulation of the SO nerve discharge usually occurred at a current of $\pm 30 \mu \mathrm{A}$, as indicated by the averaged responses over a single cycle (blue curve in Fig. $4 F)$. With larger current amplitudes, the average peak firing rate of the modulated multiunit spike activity increased and saturated at $\sim 220$ spikes/s at current amplitudes above $\pm 200 \mu \mathrm{A}$ (red symbols in Fig. 4G). Although the overall dependency of GVSinduced responses on current intensity was similar for the LR and SO nerves, the modulation threshold and saturation of the peak firing rates required larger currents for SO compared with LR nerve responses (cf. green and red symbols in Fig. $4 G$ ). This difference is likely due to the topographic relation between electrode tip and sensory epithelium that allows a closer placement of the stimulus electrode to the $\mathrm{HC}$ than the PC or AC sensory epithelia.

The dynamics of GVS-induced extraocular motor responses was characterized by modulating the LR and SO nerve activity with sinusoidal currents over a frequency range of $0.05-10 \mathrm{~Hz}$ $(n=12$; Fig. $4 H$ ). At GVS frequencies up to $\sim 1 \mathrm{~Hz}$, the peak discharge of the modulated LR nerve responses remained relatively synchronized (black and red traces in Fig. $4 H$ ) and coincided with the GVS half cycle that depolarized the HCc (Fig. 4I). Averaging both LR and SO nerve responses over a single cycle, respectively, revealed that, at stimulus frequencies $>1 \mathrm{~Hz}$, the modulated discharge became more and more asynchronous, resulting in a decrease of the peak firing rate of the modulated extraocular motor discharge (green and blue traces/curves in Fig. $4 H$, I; black symbols in Fig. $4 J$ ). This decrease in peak firing rate with increasing GVS frequency was accompanied by a concurrent shift in the timing of the response peak (red symbols in Fig. $4 J$ ). At stimulus frequencies up to $1 \mathrm{~Hz}$, responses were phase advanced by $15-30^{\circ}$, whereas, at stimulus frequencies of $>1 \mathrm{~Hz}, \mathrm{LR}$ and $\mathrm{SO}$ response peaks became more and more phase lagged, reaching $-90^{\circ}$ at a stimulus frequency of $10 \mathrm{~Hz}$ (Fig. $4 \mathrm{I}$, red symbols in $J$ ).

\section{GVS-induced extraocular motor discharge}

Independent control of bilateral push-pull inputs

The synaptic connectivity underlying the angular VOR includes for each set of extraocular motoneurons a reciprocal crossed excitatory and uncrossed inhibitory input from bilateral semicircular canal pairs coaligned with the pulling direction of the respective eye muscles (Graf and Simpson, 1981). Accordingly, the extraocular motor response modulation during sinusoidal head rotation consists of an alternating increase and decrease of the spontaneous motoneuronal discharge (Straka and Dieringer, 2004). Therefore, we next attempted to influence the two components of GVS-induced extraocular motor responses separately. This was achieved by independently altering the position of the two GVS electrodes $(n=6)$ that provoked the semicircular canalrelated excitatory and inhibitory responses, respectively (Fig. $5 A$ ). Maximal modulation of SO nerve responses evoked at a stimulus intensity of $\pm 100 \mu \mathrm{A}$ was obtained when the two GVS electrodes were placed in close proximity $(0 \mathrm{~mm}$ in Fig. $5 A)$ to the epithelia of the PCi and ACc, respectively (gray trace in Fig. $5 B$ ). Retraction of the ACc electrode to a distance of $2 \mathrm{~mm}$ from the epithelium while maintaining the second electrode close to the PCi epithelium (Fig. 5A) caused a visible reduction of the inhibitory component (blue trace in Fig. $5 B$ ). In contrast, retraction of the PCi electrode to a distance of $2 \mathrm{~mm}$ from the epithelium while maintaining the second electrode close to the ACc epithelium (Fig. 5A) caused an obvious decrease of the peak discharge that reflects the excitatory VOR component (red trace in Fig. $5 B$ ). The independent reciprocal diminishment of the two components (red and blue asterisks in Fig. 5C) became particularly evident when averaging the modulated responses over a single cycle (Fig. $5 C$ ). This independent reduction of excitatory and inhibitory components, respectively, was gradual and correlated in magnitude with the distance between stimulus electrode and epithelium (red and blue asterisks in Fig. 5D). At the most distant position of the stimulus electrodes from the epithelium (2 mm), the amplitudes of the two components, respectively, reached the level of the spontaneous resting rate (gray bar in Fig. $5 E$ ). Therefore, the systematic manipulation of the positions of the two GVS electrodes allowed an independent control of excitatory and inhibitory extraocular motor responses from particular bilateral semicircular canal pairs.

\section{Spatial specificity of stimulation sites}

As another primary purpose of the comparison between motionand GVS-evoked responses, we evaluated whether GVS through carefully placed electrodes on bilateral coplanar semicircular pairs evokes vestibulo-ocular responses with the same spatial specificity as during a rotation along the same canal plane. Accordingly, stimulus electrodes were repositioned systematically relative to the semicircular canal epithelia along the lateral aspect of the otic capsule $(n=10$; Fig. $5 F, H)$. Placement of the two GVS electrodes in close proximity of the bilateral $\mathrm{HC}$ (position 1 in Fig. 5F; stimulus intensity: $\pm 50 \mu \mathrm{A}$ ) or PCi/ACc epithelia (position 1 in Fig. $5 H$; stimulus intensity: $\pm 100 \mu \mathrm{A}$ ) provoked a maximal extraocular motor discharge modulation in the LR (trace 1 in Fig. 5G) and SO nerves, respectively, by the applied sinusoidal current (red and green curves in Fig. 5I). Simultaneous symmetric repositioning of the two $\mathrm{HC}$ electrodes caudally toward the $\mathrm{PC}$ epithelia (bilateral positions 2 and 3 in Fig. $5 F$ ) caused a gradual reduction of the LR nerve discharge modulation (traces 2 and 3 in Fig. $5 G$; green curves in Fig. $5 \mathrm{~J}, K$ ). A similar repositioning of the bilateral PCi/ACc stimulation electrodes to the reciprocal stimulus sites [i.e., ipsilateral AC (ACi)/PCc; bilateral positions 2 and 3 in Fig. $5 \mathrm{H}$ ] caused a correspondingly diminished discharge modulation of the SO nerve activity (red curves in Fig. $5 J, K$ ). This stimulus-site-specific alteration of the discharge modulation of the LR and SO nerves was further confirmed by simultaneous recordings of the two nerves during GVS $(0.5 \mathrm{~Hz} ; \pm 50 \mu \mathrm{A})$ of the bilateral HC cupulae $(n=4$; Fig. $5 L)$. At variance with the clear discharge modulation of the LR nerve (top trace in Fig. $5 L$ ), the SO nerve discharge did not display any modulation (bottom trace in Fig. $5 L$ ) up to a stimulus intensity of about $\pm 150 \mu \mathrm{A}$, corroborating a semicircular canal-specific activation of extraocular motor nerves at low to moderate stimulus intensities.

Central to the GVS-induced cyclic modulation of extraocular motor discharge and the imitation of a sinusoidal head rotation is the application of sine wave currents with oppositely oriented polarities of the bilaterally positioned electrodes. This arrangement causes activation on one side and disfacilitation of peripheral neuronal elements on the other side of, for example, the bilateral $\mathrm{HC}$ that was comparable to the situation during horizontal head rotations. This assumption was further tested by comparing LR nerve responses that were evoked by GVS of the bilateral HC (Fig. $5 \mathrm{M}, N$ ) with either opposite phase (out of phase; top trace in Fig. 5M) or with phase alignment (in phase; bottom trace in Fig. $5 M$ ) of the sinusoidal current applied to the two stimulus electrodes. At variance with the clear discharge modulation during out-of-phase stimulation (black curve in Fig. $5 N ; n=7)$, GVS with an in-phase relation of the cyclic currents (cf. green stimulus traces in top and bottom plots of Fig. 5M) did not provoke a modulation of the LR nerve activity (red curve in 
A
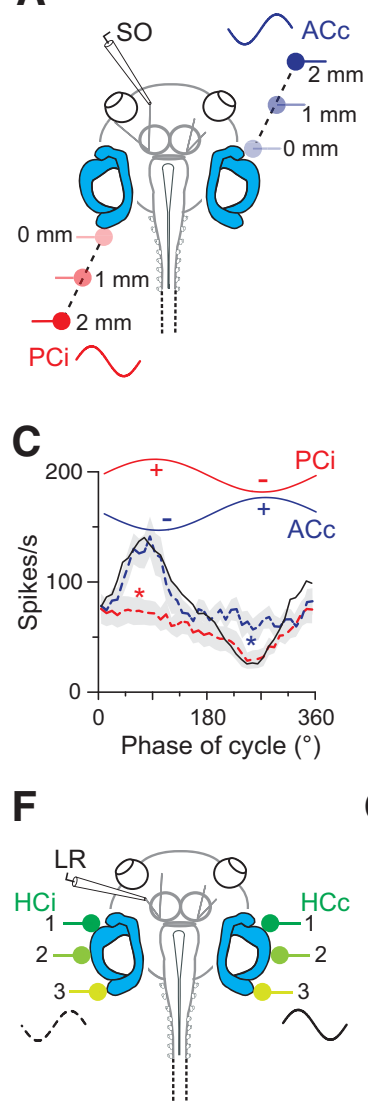

F
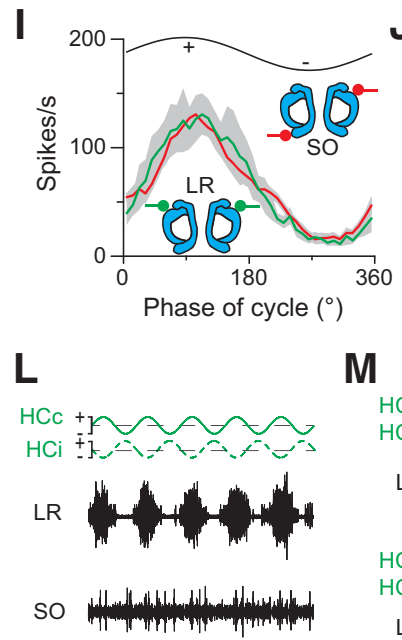

D

G

M
B

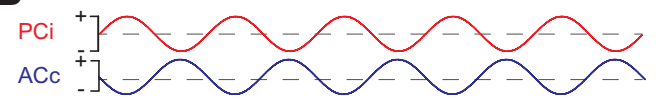

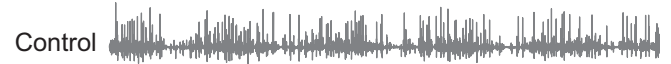
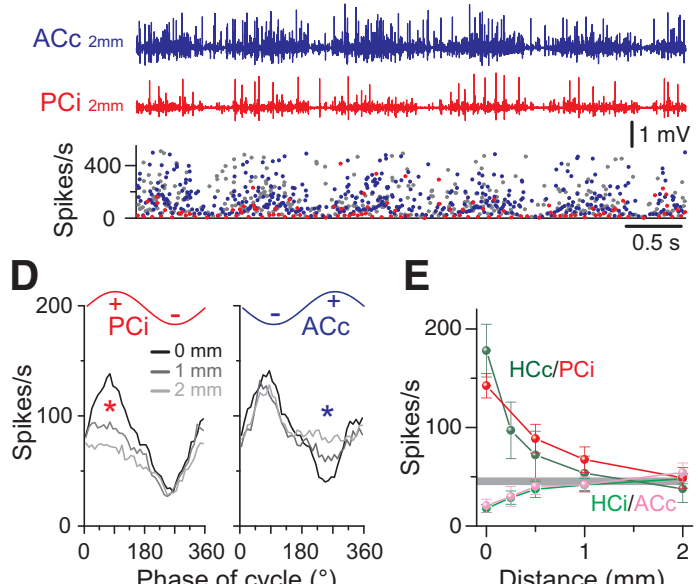

E


J
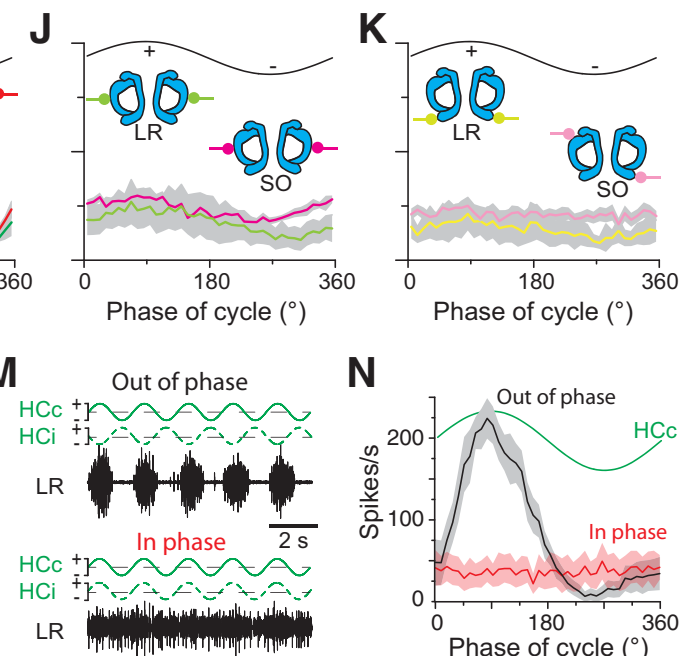

N

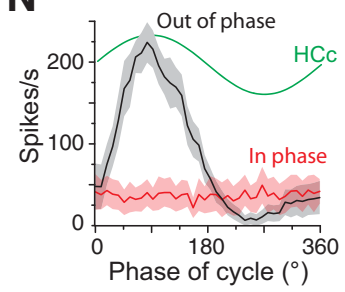

Figure 5. Stimulus site dependency of GVS-induced multiunit discharge modulation in extraocular motor nerves. $\boldsymbol{A}$, Extracellular recordings of the $S 0$ nerve during GVS of the left PC (PCi) and right $A C(A C)$ cupulae at different distances from the sensory epithelia (color-coded stimulus electrodes). $\boldsymbol{B}$, Left SO nerve discharge (middle traces) and instantaneous firing rate (bottom plot) during sinusoidal GVS of the left PC and right AC cupulae (traces in top row), with both electrodes close to the respective cupula (gray trace) and after independent repositioning of the ACc (blue trace) or PCi electrode (red trace) to a distance of $2 \mathrm{~mm}$ from the respective cupulae. $\boldsymbol{C}$, Averaged responses over a single GVS cycle (from 16 cycles; \pm SEM, gray shaded areas; $n=6$ ) with both stimulus electrodes close to the cupulae (black trace) and with the ACc (blue dashed trace) or PCi electrode (red dashed trace) at a distance of $2 \mathrm{~mm}$ from the cupula; Note the absence of either the inhibitory (blue asterisk) or the excitatory component (red asterisk) under the latter two stimulus conditions. D, Averaged responses over a single GVS cycle (from 16 cycles in $n=6$ preparations) with the $\mathrm{PCi}$ or ACc electrode at increasing distances from the epithelium; note the gradual reduction of excitatory (red asterisk) and inhibitory components (blue asterisk), respectively. $\boldsymbol{E}$, Dependency of HCC/PCi-evoked excitatory and HCi/ACCevoked inhibitory response components in the LR and SO nerves on electrode position; the horizontal gray bar indicates the mean \pm SEM of the $L R / S O$ resting rates. $\boldsymbol{F}, \boldsymbol{H}$, Recordings of the $L R(\boldsymbol{F})$ and SO nerve $(\boldsymbol{H})$ during GVS of the HCc/HCi and the PCi/ACc cupulae and after bilateral electrode repositioning ( $n=10$; color-coded electrodes 1-3 in $\boldsymbol{F}, \boldsymbol{H})$. $\boldsymbol{G}$, Left LR nerve discharge (color-coded traces) and instantaneous rate (bottom plot) during sinusoidal GVS of the HCC/HCi cupulae (traces in top row) at three

Fig. $5 N ; n=7)$. This lack of modulation is compatible with a concurrent activation of excitatory and inhibitory vestibulo-ocular connections when the semicircular canal sensory peripheries on both sides are simultaneously activated/disfacilitated.

The systematic reduction of cyclic LR and SO nerve activity by altering the distances of the stimulus electrodes from the respective sensory epithelia in a stepwise manner indicates that the optimal semicircular canal position has a high specificity for the electrical activation of the three-neuronal VOR with respect to a particular spatial plane. Although the stimulus-site-specific alterations are consistent with the spatially limited extension of the electric field for GVS (Fig. 1G,H), this specificity also complies with the conserved main VOR connections between particular semicircular canals and sets of extraocular motoneurons (Precht, 1978; Straka and Dieringer, 2004).

\section{GVS-induced modulation of $\mathrm{Ca}^{2+}$} transients in central vestibular neurons The pattern of GVS-induced neuronal activity was studied by $\mathrm{Ca}^{2+}$ imaging of identified vestibulo-ocular projection neurons (green fibers and cell bodies in Fig. 6A) labeled retrogradely with a $\mathrm{Ca}^{2+}$ sensor (Calcium Green-1 dextran) from the oculomotor nucleus. With respect to the injection site, clusters of oculomotornucleus-projecting VOR neurons were consistently labeled ipsilateral in rhombomeres (r) 2-3 and contralateral in r5-6 (white outlined areas in Fig. 6A), compatible with earlier studies (Straka et al., 2001, 2002a). The two populations coincide with the known segmental positions of VOR neurons that receive excitatory vestibular afferent inputs from an ipsilateral vertical semicircular canal (Straka et

\section{$\leftarrow$}

stimulus electrode positions (1-3, defined in $\boldsymbol{F})$. $\boldsymbol{I}-\boldsymbol{K}$, Averaged responses of the $L R$ and $S O$ nerves over a single GVS cycle (from 16 cycles, \pm SEM, gray shaded areas; $n=10$ ) evoked with both electrodes close to the cupulae $(\mathrm{HCC} / \mathrm{HCi}, \mathrm{PCi} / \mathrm{ACc}$ in I) and after bilateral repositioning of the electrodes ( $\boldsymbol{J}, \boldsymbol{K}$; colorcoded electrodes also in $\boldsymbol{F}, \boldsymbol{H})$. $\boldsymbol{L}$, Simultaneous recordings of the left LR (top trace) and SO (bottom trace) nerve during 0.5 $\mathrm{Hz}$ sinusoidal GVS $( \pm 50 \mu \mathrm{A})$ of the bilateral HC cupulae. $\mathbf{M}, \mathbf{N}$, LR nerve discharge during sinusoidal GVS $(0.5 \mathrm{~Hz} ; \pm 100 \mu \mathrm{A})$ of the bilateral $\mathrm{HC}$ cupulae with sinusoids that polarized the two stimulus electrodes either in phase opposition (out of phase; top traces in $\boldsymbol{M}$ ) or in phase alignment (in phase; bottom traces in $\boldsymbol{M})$; averaged extraocular motor responses $(\boldsymbol{N}$, $n=7$ ) over a single GVS cycle at $0.5 \mathrm{~Hz}$ (from 16 cycles, \pm SEM, gray and light red shaded areas) with out-of-phase (black curve) or in-phase (red curve) polarization. Scale bar in $\boldsymbol{M}$ applies also to $L$. 

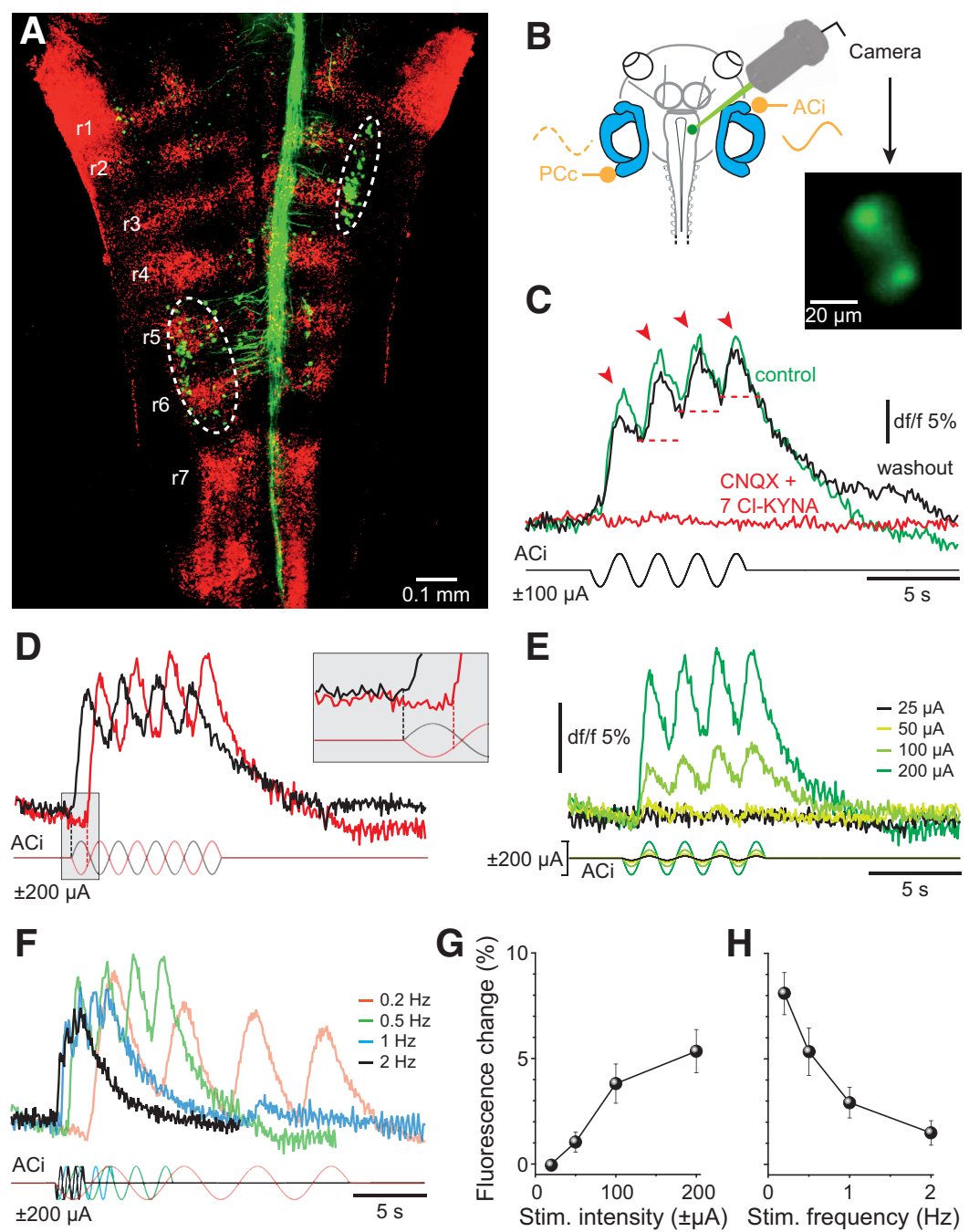

Figure 6. GVS-induced calcium dynamics in vestibulo-ocular neurons. $\boldsymbol{A}$, Confocal reconstruction of hindbrain whole mounts after application of Alexa Fluor 488 dextran to the right oculomotor nucleus depicting retrogradely labeled vestibulo-0cular projection neurons (green) in ipsilateral r2-3 and contralateral r5-6 (white encircled areas); rhombomeres (red) were visualized with 633 nm illumination. $\boldsymbol{B}$, Recording of $\mathrm{Ca}^{2+}$ transients in $\mathrm{r2}-3$ vestibulo-ocular neurons (green neurons in inset) during GVS of the ACi and PCc; neurons were retrogradely labeled with the $\mathrm{Ca}^{2+}$-sensor (Calcium Green-1 dextran) from the ipsilateral oculomotor nucleus. $C$, GVS-induced $\mathrm{Ca}^{2+}$ transients before (green trace, control), during (red trace), and after 30 min of wash-out (black trace) of CNQX (15 $\mu \mathrm{m})$ and 7-Cl-KYNA $(50 \mu \mathrm{m})$; arrowheads indicate cyclic phase-timed fluorescence peaks; red dashed lines indicate successively elevated $\mathrm{Ca}^{2+}$ levels. $\boldsymbol{D}-\boldsymbol{F}, \mathrm{Ca}^{2+}$ transients induced by GVS with opposite stimulus polarities (red and black stimulus traces, $\boldsymbol{D}$ ), increasing current stimulus amplitudes (color-coded traces, $\boldsymbol{E}$ ) and increasing stimulus frequency (color-coded traces, $\boldsymbol{F}$ ). Inset in $\boldsymbol{D}$ illustrates a half-cycle shift in response onset with inversion of the stimulus polarity. $\boldsymbol{G}, \boldsymbol{H}$, Dependency of fluorescence peak amplitude $(\boldsymbol{G})$ and cyclic fluorescence oscillations $(\boldsymbol{H})$ on stimulus intensity and frequency, respectively. Fluorescence calibration ( $\mathrm{d} F / F$ ) in $\boldsymbol{E}$ also applies to $\boldsymbol{D}$ and $\boldsymbol{F}$.

al., 2002b) and commissural inhibitory inputs from the corresponding contralateral coplanar semicircular canal (Holler and Straka, 2001). Accordingly, we simultaneously stimulated the ipsilateral AC and contralateral PC with sinusoidal GVS (Fig. 6B) and recorded $\mathrm{Ca}^{2+}$ transients of identified VOR neurons in $\mathrm{r} 2-3$ that were backfilled with the $\mathrm{Ca}^{2+}$ sensor (green neurons in Fig. $6 B$ ). The majority of these neurons (12 of 16 neurons in five preparations) exhibited fluorescence changes during GVS (Fig. $6 C)$. At a stimulus frequency of $0.5 \mathrm{~Hz}$, these $\mathrm{Ca}^{2+}$ responses consisted of cyclic fluorescence oscillations (red arrowheads in Fig. $6 C$ ), with the peaks being phase timed to the depolarization of the ipsilateral AC. During the alternating hyperpolarization, the fluorescence failed to reach the initial baseline, likely due to the low dynamics of the $\mathrm{Ca}^{2+}$ sensor, thus causing each subse- quent transient to start from a successively higher $\mathrm{Ca}^{2+}$ level (red dashed lines in Fig. 6C).

To demonstrate that the observed GVSinduced $\mathrm{Ca}^{2+}$-responses were mediated synaptically, rather than by direct electrical activation of central vestibular neurons, we pharmacologically blocked the glutamatergic transmission between vestibular nerve afferents and second-order vestibular neurons (Fig. 6C). Combined bath application of the AMPA receptor blocker CNQX (15 $\mu \mathrm{M}$ ) and NMDA blocker 7-Cl-KYNA (50 $\mu \mathrm{M})$ reversibly abolished the stimulusevoked fluorescence modulation (red trace in Fig. $6 C$ ), thus excluding a direct activation of central vestibular neurons by GVS. Accordingly, $\mathrm{Ca}^{2+}$ transients in VOR neurons were provoked by vestibular nerve afferent discharge after activation of cellular elements in the semicircular canal epithelium. However, this activation exhibited a distinct specificity with respect to the polarity of the galvanic stimulus. In fact, $\mathrm{Ca}^{2+}$ transients of imaged VOR neurons were only activated by cathodal currents at the electrode close to the ipsilateral AC cupula (Fig. 6D). Accordingly, after inversion of the stimulus polarity, the response onset was shifted by a half cycle, coinciding again with a depolarization of the ACi epithelium (compare red and black traces in inset of Fig. 6D).

The activation of VOR neurons through bilateral GVS of the ACi/PCc was further evaluated by systematically altering stimulus amplitude and frequency (Fig. 6E,F). With increasing GVS magnitude $( \pm 25-200 \mu \mathrm{A})$, the amplitude of the fluorescence peaks (Fig. 6E, G), as well as the fluorescence level from which the $\mathrm{Ca}^{2+}$ transients started during each successive depolarizing half cycle, increased gradually (see also red dashed lines in Fig. $6 C)$. A similar augmentation of the fluorescence level over the stimulus period was observed with increasing frequencies $(0.2-2 \mathrm{~Hz})$, whereas, at the same time, the cyclic-phase-timed fluorescence peaks became gradually smaller and less discernible (Fig. 6F,H). This latter decline is again likely due to the slow decay time constant of the evoked $\mathrm{Ca}^{2+}$-responses that restricts a dissociation of individual peaks with increasing GVS frequencies. Nonetheless, individual $\mathrm{Ca}^{2+}$ oscillations were still distinguishable up to a stimulus frequency of $2 \mathrm{~Hz}$ (Fig. $6 H$ ).

\section{GVS-induced modulation of vestibular nerve afferent discharge}

GVS-induced extraocular motor activity or discharge of VOR neurons depends on the activation of excitable cells in the vestibular sensory epithelium. Because the membrane potential of both hair cells and afferent fibers is sensitive to electrical currents, GVS could either trigger action potentials in vestibular afferents di- 
rectly or indirectly through hair cell depolarization and increased transmitter release or recruit both cellular substrates. To differentiate between these possibilities, we recorded multiunit spike discharge from the anterior branch of the VIII ${ }^{\text {th }}$ nerve and the spike activity of single afferent nerve fibers during sinusoidal rotation and GVS of the HC or AC sensory epithelium (Fig. 7A). During sinusoidal turntable rotation, the multiunit discharge (dark red trace in Fig. $7 B$ ) and the spike activity of individual afferents (HC afferent fiber; light red trace in Fig. $7 B$ ) became cyclically modulated with an increase of the discharge during ipsiversive motion. However, independent of the number of recorded units, the peak firing rate of the multiunit recording $(n=8)$ increased gradually with peak velocity over the used stimulus range (red shaded area in Fig. $7 D$ ). This discharge profile was confirmed by single-unit $\mathrm{HC}(n=34)$ and AC $(n=5)$ nerve afferent recordings; however, as expected, single units had peak firing rates that were lower than those of the multiunit recordings [ $\mathrm{cf}$. multiunit (M) and single-unit (S) recordings in left plot of Fig. $7 C$ ]. Nonetheless, the increase in maximal firing rate with peak stimulus velocity was very similar between single-unit and multiunit recordings (cf. red symbols with red shaded area in Fig. $7 D$ ) and not significantly different from each other (Mann-Whitney $U$ test). The relatively wide range of resting rates between 0.1 and 14.1 spikes/s (mean rate \pm SD: $5.10 \pm 4.95$ spikes/s; $n=39$ ) and discharge regularity between 0.30 and 1.46 (mean CV \pm SD: $0.83 \pm$ $0.32 ; n=39$ ) of the recorded single units suggest that a relatively broad population of dynamically different vestibular nerve afferent fibers has been included in the analysis (Honrubia et al., 1989).

During GVS of the HC or AC cupula, the afferent spike discharge (HC singleunit: green traces; multiunit: blue traces in Fig. $7 B$ ) was sinusoidally modulated with a similar difference in peak firing rates between multiunit and single-unit recordings (cf. M and S in right plot of Fig. $7 C$ ) as seen for the corresponding responses during rotational stimuli (left plot of Fig. 7C). With increasing GVS currents, the peak firing rate increased for both multiunit recordings (blue traces in Fig. $7 B$ ) and single-unit recordings (green traces in Fig. $7 B$ ). In fact, the maximal firing rate augmented similarly with current intensity in both cases (cf. green symbols with green shaded area in Fig. $7 E$ ) and was not significantly different from each other (MannWhitney $U$ test). The relatively linear correlation between vestibular afferent peak firing rate and stimulus intensity over a wide range during rotation and GVS, respectively (Fig. 7D,E), allowed calibrating the GVS current with respect to the motion stimulus amplitude (red and black line in Fig. $7 F$ ). Using the peak dis-
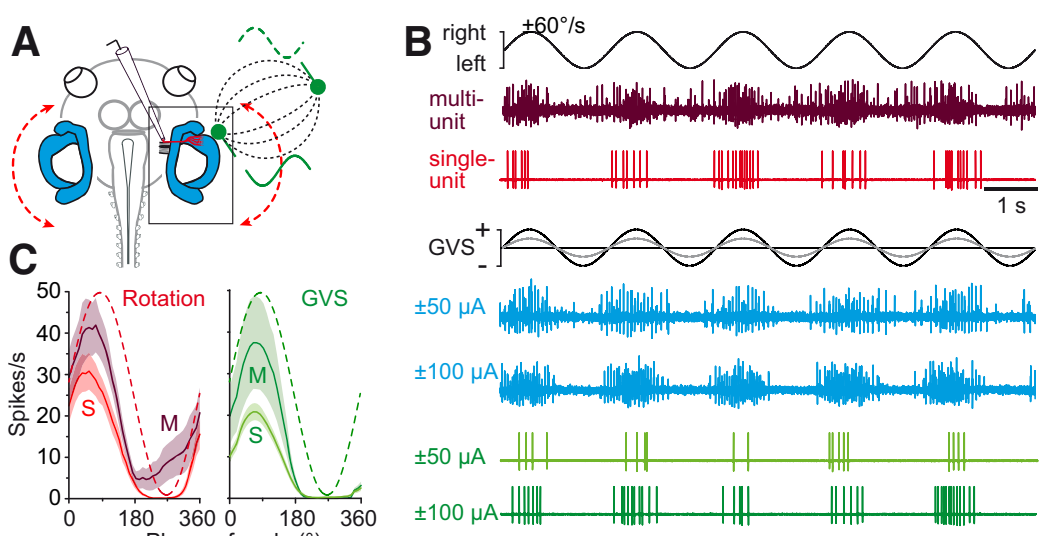



E
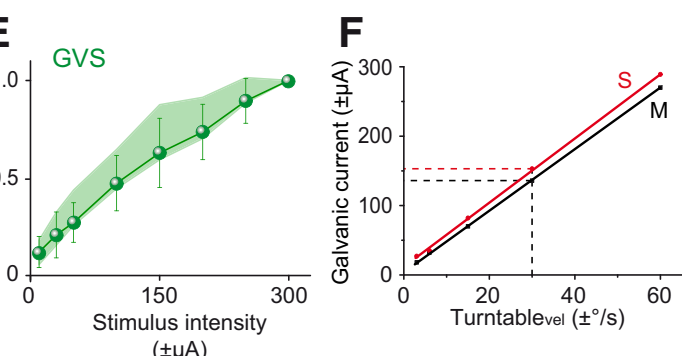

H

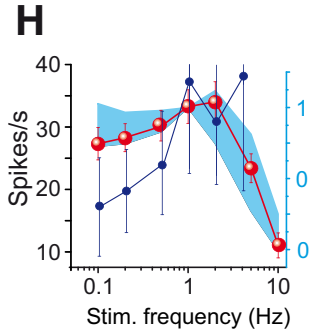

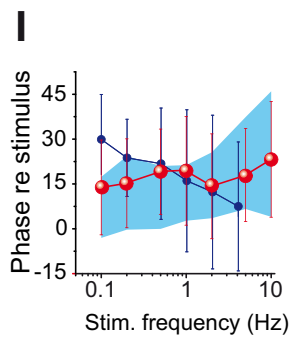

Figure 7. Dynamics of rotation- and GVS-evoked HC afferent neuronal discharge. $\boldsymbol{A}$, Extracellular recordings of afferent fibers in the anterior branch of the right VIII ${ }^{\text {th }}$ nerve during horizontal rotation (red dashed curve) and GVS of the right HC cupula (green electrode); note the electric field (black dashed field lines) between the $\mathrm{HC}$ and a second electrode in the bath solution (green electrode). $\boldsymbol{B}$, Multiunit discharge (dark red trace; blue traces) and HC single-unit discharge (light red trace; green traces) during

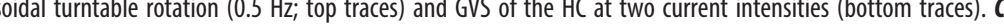
(from 16 -SEM, shaded areas); red and green dashed sinusoids indicate the current stimulus. $\boldsymbol{D}, \boldsymbol{E}$, Dependency of the normalized firing rates on rotation $(\boldsymbol{D})$ and GVS stimulus intensities $(\boldsymbol{E})$. $\boldsymbol{F}$, Calibration of the GVS with respect to turntable peak velocity based on corresponding multiunit ( $\mathrm{M}$, black line) and single-unit ( $\mathrm{S}$, red line) firing rates; imitation of $30^{\circ}$ /s turntable peak velocity (at 0.5 $\mathrm{Hz}$ ) requires GVS currents of $\pm 140 \mu \mathrm{A}$ and $\pm 155 \mu \mathrm{A}$, respectively (dashed lines). G, HC single-unit discharge during sinusoidal GVS $( \pm 100 \mu \mathrm{A})$ of the $\mathrm{HC}$ at 4 stimulus frequencies $(n=5)$. $\boldsymbol{H}, \boldsymbol{I}$, Average amplitude $(\boldsymbol{H})$ and phase $(\boldsymbol{I})$ of multiunit responses $n=20$ ) and sinusoidal rotation (dark blue symbols in $\boldsymbol{H}, \boldsymbol{I} ; \boldsymbol{n}=26$ ) at different stimulus frequencies; note that multiunit firing rates were normalized in $\boldsymbol{H}$. Scale bar in $\boldsymbol{B}$ applies to all traces; calibration bar in $\mathbf{G}$ for $0.2 \mathrm{~Hz}$ applies also to $1 \mathrm{~Hz}$.

charge of vestibular nerve afferents obtained from single-unit ( $\mathrm{S}$, red line) and multiunit ( $\mathrm{M}$, black line) recordings during rotation and GVS as common parameter, this comparison allowed translating peak head velocity into GVS current amplitude and vice versa. Given the matching dependency of single-unit and multiunit discharge on stimulus intensities, respectively (Fig. $7 D, E$ ), the calibration yielded similar results in both cases (red and black lines in Fig. $7 F$ ). Accordingly, imitation of the discharge during a sinusoidal rotation with a peak velocity of $30 \%$ requires a GVS current of approximately $\pm 150 \mu \mathrm{A}$.

Whereas multiunit and single-unit recordings revealed similar afferent response dynamics, the multiunit recording from the anterior branch of the VIII ${ }^{\text {th }}$ nerve captured the activity of a rather heterogeneous group of fibers that very likely also included 

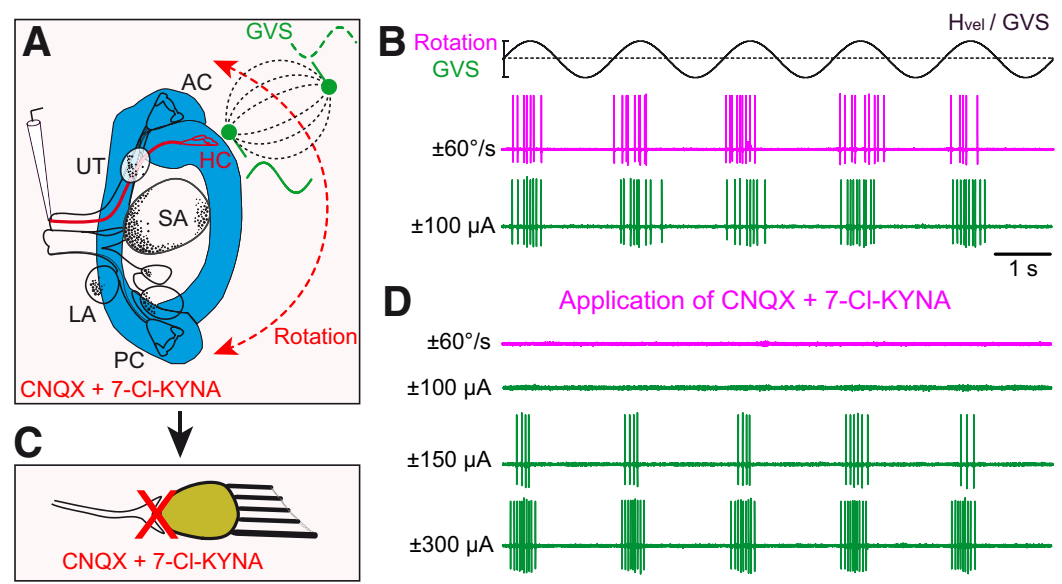

D
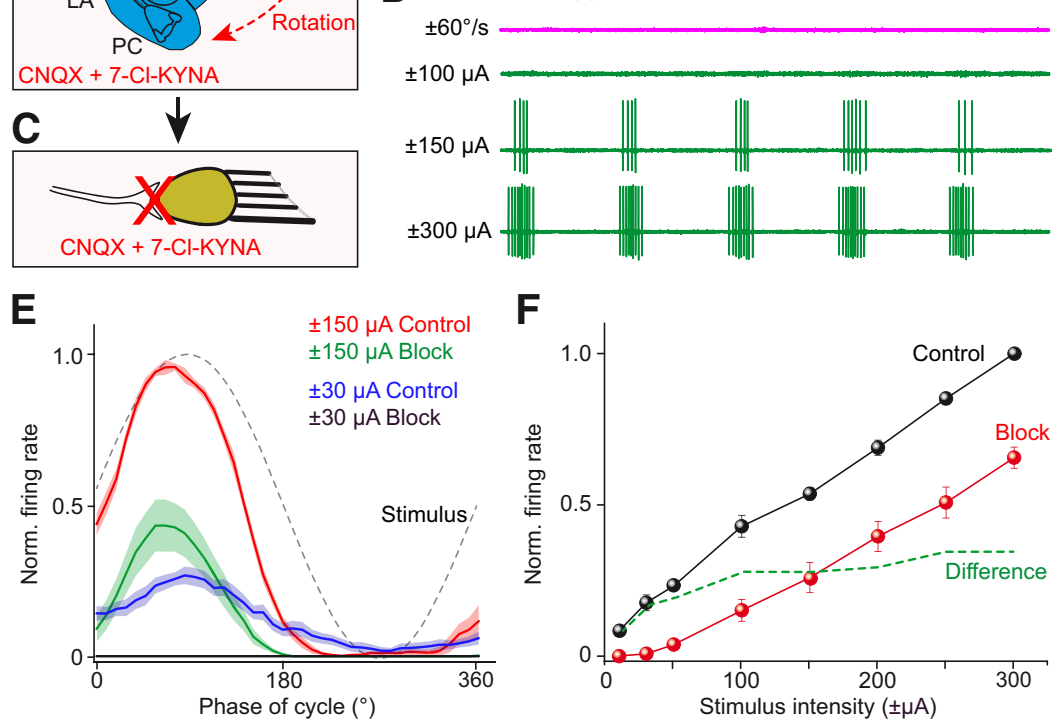

Figure 8. Determination of the cellular substrate underlying GVS-induced spike discharge in vestibular nerve afferents. $\boldsymbol{A}$, Single-unit recordings of $\mathrm{HC}$ afferents during horizontal turntable rotation (red dashed arrow) and GVS of the HC cupula (green stimulus electrode); note that the GVS electric field (dashed field lines) expands between the HC electrode and a distant one in the Ringer's solution. $\boldsymbol{B}-\boldsymbol{D}$, Single HC afferent fiber discharge during horizontal rotation (magenta traces in $\boldsymbol{B}, \boldsymbol{D}$ ) and GVS of the HC cupula (green traces in $\boldsymbol{B}, \boldsymbol{D})$ before $(\boldsymbol{B})$ and during $(\boldsymbol{D})$ bath application of CNQX (15 $\mu \mathrm{m}$ ) and 7-CI-KYNA (50 $\mu \mathrm{m}$ ) that blocked the synaptic transmission between hair cells and vestibular afferent fibers pharmacologically (C). $\boldsymbol{E}, \boldsymbol{F}$, Averaged single-unit afferent discharge over one cycle of GVS (from 16 cycles, \pm SEM, shaded areas; $n=13$ ) at two stimulus intensities ( $\pm 30 \mu A, \pm 150 \mu \mathrm{A}$; $\boldsymbol{E}$ ) and dependency of GVS-induced peak afferent firing rate on stimulus intensity $(\boldsymbol{F})$ before (control, black symbols) and during pharmacological block of the glutamatergic hair cell-afferent synapse (red symbols); green dashed line in $\boldsymbol{F}$ depicts the arithmetic difference between the two conditions, indicating the magnitude of hair cell contribution to the GVS-induced afferent discharge. Scale bar in $\boldsymbol{B}$ applies to all traces in $\boldsymbol{B}$ and $\boldsymbol{D}$.

utricular afferents along with $\mathrm{HC}$ and AC afferents. To obtain a more homogeneous population of afferent fibers for further analysis, we restricted our sampling to identified $\mathrm{HC}$ and $\mathrm{AC}$ single units $(n=26)$. GVS of the HC or AC at different stimulus frequencies $(0.1-10 \mathrm{~Hz})$ caused an afferent discharge modulation (Fig. $7 G$ ) with a maximal peak discharge at a stimulus frequency of $\sim 1-2 \mathrm{~Hz}$ (red symbols in Fig. $7 \mathrm{H}$ ), which was similar to that of multiunit recordings (blue area in Fig. $7 H$ ). This pattern is similar to that of motion-induced responses in the same afferent population with the exception of a further increase in sensitivity above a motion frequency of $1 \mathrm{~Hz}$ (dark blue symbols in Fig. 7H) and generally complies with the dynamics of mammalian afferent fibers despite their separation into different subtypes (Kim et al., 2011). The concurrent phase lead of GVS-induced responses with respect to stimulus velocity in the range of $10-20^{\circ}$ at $0.1 \mathrm{~Hz}$ was relatively constant over the tested range of stimulus frequencies for both single-unit responses (red symbols in Fig. 7I) and multiunit responses (blue area in Fig. 7I). This phase independence was similar to the phase relation of the responses during sinusoidal head motion (dark blue symbols in Fig. 7I), which, however, in the current study could only be evaluated up to a stimulus frequency of $4 \mathrm{~Hz}$ due to the impaired stability of the afferent recordings at even higher stimulus frequencies. Unfortu- nately, this prevented a direct comparison of phase values of GVS- and motioninduced responses at higher frequencies as in mammals (Kim et al., 2011). In the current study, the broad distribution of amplitude modulation and phase values over the range of used frequencies is likely related to the relatively broad spectrum of recorded single units, as indicated by the range of resting rates and CVs (see above).

\section{Cellular substrate for galvanic stimulation of semicircular canals}

To resolve the question of which cellular substrate(s), hair cells and/or vestibular nerve afferents are activated by GVS, we recorded the galvanically induced discharge modulation of $\mathrm{HC}$ and $\mathrm{AC}$ afferent fibers $(n=13)$ during pharmacological blockade of the synaptic transmission between hair cells and vestibular afferent fibers (Fig. $8 A, C$ ). All recorded afferent fibers were robustly modulated during either horizontal rotation ( $\mathrm{HC}$ afferents) or rotation in the direction of the ipsilateral AC (AC afferents), as well as during GVS before drug application (magenta and green traces in Fig. 8B). Combined bath application of AMPA receptor (CNQX: 15 $\mu \mathrm{M})$ and NMDA receptor (7-Cl-KYNA: $50 \mu \mathrm{M})$ antagonists completely blocked the motion-induced discharge modulation (magenta trace in Fig. 8D), confirming the suppression of the glutamatergic transmission between hair cells and vestibular nerve afferents (Fig. 8C). Similarly, the discharge modulation induced by low GVS intensities was blocked under this condition (cf. green traces at a GVS of $\pm 100 \mu \mathrm{A}$ in Fig. 8 B, D). However, the discharge modulation evoked by higher GVS currents persisted (green traces at a GVS of \pm 150 and $300 \mu \mathrm{A}$ in Fig. $8 D$ ), even though peak firing rates were markedly reduced (Fig. $8 E, F$ ). The failure to induce a discharge modulation by GVS at stimulus intensities $<50 \mu \mathrm{A}$ (Fig. $8 F$ ) in the presence of glutamatergic antagonists suggests a preferential recruitment of hair cells at low stimulus intensities, with only limited increase at higher currents (dashed line in Fig. 8F). Therefore, the partial reduction of GVSinduced vestibular afferent discharge modulation, when the hair cell-afferent synaptic transmission was blocked (Fig. $8 E, F$ ), indicates that, under control conditions, both hair cells and afferent fibers are recruited by galvanic vestibular stimulation even though a direct activation of vestibular afferents predominated at higher stimulus intensities.

\section{Discussion}

Sinusoidal galvanic stimulation of semicircular canals provokes a modulation of neuronal activity in all VOR elements. The GVS site dependency of response magnitudes and matching spatiotemporal profiles during GVS and rotation demonstrate that applied currents activate canal-specific circuits and imitate natural head movements over a broad range of frequencies and amplitudes. The partially reduced GVS-evoked vestibular afferent dis- 
charge following a block of the glutamatergic transmission indicates that galvanic currents recruit both hair cells and vestibular nerve afferent fibers.

\section{Cellular substrate of GVS-evoked responses}

Clinical interpretation of galvanically evoked responses in patients with inner ear pathologies depends on the knowledge of the cellular substrate that is activated by GVS. Ever since the study by Goldberg et al. (1984), the relatively unchallenged notion was that GVS activates vestibular nerve afferents directly at the action potential trigger site, bypassing hair cell synapses (see Fitzpatrick and Day, 2004). Based on axon diameters, cathodal and anodal galvanic currents predominantly recruit and silence irregular afferent fibers, respectively, with a limited influence on regularly firing afferents at higher stimulus intensities (Ezure et al., 1983; Goldberg et al., 1984; Kim and Curthoys, 2004; Kim et al., 2011; Shanidze et al., 2012). However, until now, no direct experimental demonstration was available showing that GVS-evoked responses in vestibulo-motor circuitries are activated without hair cell contribution. Nonetheless, the view that vestibular afferents are the exclusive origin of GVS-induced vestibular responses still prevails. Recently, however, electrical induction of VORs in gentamicin-treated human patients has challenged this assumption (Aw et al., 2008). The impairment of evoked eye movements in the latter study was interpreted as evidence that GVS-induced responses depend, at least in part, on the electrical recruitment of hair cells (Aw et al., 2008).

A significant hair cell contribution to GVS-induced afferent discharge was in fact confirmed in the present study (Fig. 8). After complete pharmacological blockade of the glutamatergic transmission between hair cells and afferent fibers, GVS-induced responses persisted, although at reduced magnitudes (Fig. $8 F$ ). The loss of part of the GVS-induced afferent activity suggests that a direct activation of hair cells normally contributes to the discharge modulation, a result that complies with the findings in patients with gentamicin-induced vestibular deficits (Aw et al., 2008). Whereas our approach benefited from spatially specific locations of stimulation electrodes and preservation of intact inner ears, an activation of both peripheral excitable elements in other species or with other electrode placements is likely and might finally reconcile previously opposing views on the cellular substrate of GVS. This general extension of our finding is also supported by very similar species-independent dynamics of GVS-induced vestibular afferent responses (Ezure et al., 1983; Kim et al., 2011) and by the overall evolutionary conservation of the vertebrate inner ear (Goldberg, 2000; Fritzsch and Straka, 2014).

\section{Spatial specificity of vestibular end-organ activation by GVS}

Placement of GVS electrodes outside the intact otic capsule in close proximity to the semicircular canal cupulae in the present study was facilitated by the plain visibility of the inner ear in $X$. laevis tadpoles (Straka and Simmers, 2012). This experimental condition has the advantage of leaving endolymph motiondriven cupula displacements unaffected. The locally restricted electric field (Fig. 1G) and the stimulus site dependency of responses (Fig. 5C, D, $I-K$ ) indicate that single or pairs of bilateral coplanar semicircular canals, and thus plane-specific vestibulomotor pathways, can be activated separately, compatible with the effects of a multichannel vestibular prosthesis in monkeys (Dai et al., 2013). This spatiotemporal specificity of GVS-evoked extraocular motor responses was validated by our experiments indicating that careful placement of the electrodes evokes responses with the same spatial specificity as during a rotation along the same canal plane. This also largely excludes concurrent electrical activation of other semicircular canals or the more distant otolith organs. Accordingly, stimulus electrode placement on a specific semicircular canal in X. laevis is more precise compared with most studies on mammalian species/humans in which electrodes were inserted into the perilymphatic space of the inner ear (Ezure et al., 1983; Kim et al., 2011; Shanidze et al., 2012), the middle ear cavity (Kim and Curthoys, 2004; Kim, 2013a, 2013b), or onto the skin (Fitzpatrick and Day, 2004) and activated all vestibular end organs, as evidenced by, for example, current-evoked eye (Schneider et al., 2002) or head (Kim, 2013a) movements.

Despite the different positions of the internally placed stimulus electrodes, relatively similar current intensities $( \pm 100 \mu \mathrm{A})$ were required to evoke responses in vestibular afferents (Kim and Curthoys, 2004; Kim et al., 2011; this study) and central vestibular pathways or to effectively trigger VOR behavior (Shanidze et al., 2012). Minor variations are likely related to size/resistance of the stimulus electrodes and/or leak currents. These values, however, differ by $\sim 10$-fold from those required for transmastoidal stimulation in human subjects (Fitzpatrick and Day, 2004). Although absolute values depend largely on the experimental approach, a comparison of motion- and GVS-induced responses allowed calibrating the current stimulus (Fitzpatrick and Day, 2004). With regard to eye movements (Fig. 2) and vestibular afferent discharge (Fig. 7) in X. laevis tadpoles, the respective general patterns and dynamics of current- and motion-induced responses match very well (Figs. $2 G, 7 F$ ). Moreover, the corresponding calibration of GVS-induced afferent discharge and eye movements indicates that a representative sample of neurons was recorded at each synaptic level of the VOR. Moreover, the similarity of responses in this study compared with other studies (Ezure et al., 1983) suggests that the interpretation of our results can be extended onto other GVS models.

\section{Dynamic properties of GVS-evoked responses}

Most previous studies used current pulses to study GVS-induced neuronal responses and vestibulo-motor behaviors or to perturb activity patterns during motion stimulation (Shanidze et al., 2012; Kim, 2013a, 2013b). In addition, current- and motionevoked responses were generally compared at one level of the VOR circuitry (Kim and Curthoys, 2004; Kim et al., 2011). In contrast, our approach, which probed each synaptic stage of the VOR, allowed estimating the dynamic similarity of motion and GVS-induced responses at multiple levels. This attempt revealed that sinusoidal GVS corresponds more closely to the velocity than the position component of head movements (Fig. 2C,D), with similar phase relations between GVS- and motion-evoked responses in VOR neuronal elements over most of the tested frequency range $(\sim 0.2-1 \mathrm{~Hz})$. The tendency of more phaseadvanced GVS-, compared with motion-induced responses $>1$ $\mathrm{Hz}$ in our study is likely due to a direct activation of vestibular afferents, which bypasses canal fluid dynamics, cilial bundle deflection, and synaptic transmission between hair cells and afferent fibers, a finding that is even more pronounced in the cat (Ezure et al., 1983) and chinchilla (Kim et al., 2011), in which higher stimulus frequencies were used. The further increasing sensitivity of afferents for motion-evoked responses $>2 \mathrm{~Hz}$ at variance with GVS-induced afferent discharge (Fig. $7 \mathrm{H}$ ) is compatible with data from the chinchilla (Kim et al., 2011) and might be related to an altered efficacy of GVS at higher frequencies. Moreover, specific differences between GVS- and rotationevoked response dynamics in mammals and amphibians might 
be related to afferent fiber types, stimulus frequencies used, and/or electrode placement. Because recruitment order and activation threshold of hair cells and afferent fibers differ between electrical and motion stimulation, any comparison between GVS- and motion-induced responses requires careful interpretation (Kim and Curthoys, 2004; Kim et al., 2011).

In contrast, differences in timing between GVS-induced extraocular motor discharge and eye movements are similar to those of respective motion-induced responses and are likely related to the additional synapse between motoneurons and muscle fibers and the buildup of muscle strength. The available response dynamics of various VOR neuronal elements in the same animal model allows determining transfer functions that might be also relevant for other species. Although GVS pulses are convenient to determine response onsets (Goldberg et al., 1984), sinusoidally oscillating stimuli appear to be more suitable for activating irregular vestibular afferents given their particularly fast response dynamics (Goldberg, 2000), which in part are due to voltagedependent $\mathrm{K}^{+}$-channels (Eatock and Songer, 2011).

The possibility of using GVS to imitate body motion will facilitate the conduction of fluorescence imaging experiments in central nervous circuits that render natural vestibular stimulation challenging. The possibility of differentiating GVS-induced responses temporally during $\mathrm{Ca}^{2+}$ imaging up to $2 \mathrm{~Hz}$ (Fig. 6) will facilitate studies that aim at understanding the organization of frequency-tuned vestibular pathways for sensory-motor transformation (Straka et al., 2005). Moreover, knowledge of the activated cellular substrates allows a more faithful categorization of vestibular disorders (Curthoys, 2010) or further improvement of vestibular prostheses (Fridman and Della Santina, 2013). In animals (Gong and Merfeld, 2000; Dai et al., 2011; Mitchell et al., 2013) and human patients (Phillips et al., 2015), vestibular prostheses provide controlled inputs that trigger vestibular reflexes (Dai et al., 2013; Lewis, 2015). Whereas prosthetic rate stimulation activates ampullary nerve fibers directly, modulated DC currents might also recruit hair cells, if intact. However, more insight into the mechanistic basis of GVS is required to understand the differential activation of excitable elements within the vestibular epithelia. Once this is resolved, GVS will considerably facilitate the diagnosis and treatment of vestibular deficits and the understanding of basic aspects of sensory-motor transformations.

\section{References}

Angelaki DE, Perachio AA (1993) Contribution of irregular semicircular canal afferents to the horizontal vestibuloocular response during constant velocity rotation. J Neurophysiol 69:996-999. Medline

Aw ST, Todd MJ, Aw GE, Weber KP, Halmagyi GM (2008) Gentamicin vestibulotoxicity impairs human electrically evoked vestibulo-ocular reflex. Neurology 71:1776-1782. CrossRef Medline

Aw ST, Aw GE, Todd MJ, Halmagyi GM (2013) Enhanced vestibulo-ocular reflex to electrical vestibular stimulation in Meniere's disease. J Assoc Res Otolaryngol 14:49-59. CrossRef Medline

Beck JC, Gilland E, Baker R, Tank DW (2004) Instrumentation for measuring oculomotor performance and plasticity in larval organisms. Methods Cell Biol 76:385-413. CrossRef Medline

Biesdorf S, Malinvaud D, Reichenberger I, Pfanzelt S, Straka H (2008) Differential inhibitory control of semicircular canal nerve afferent-evoked inputs in second-order vestibular neurons by glycinergic and GABAergic circuits. J Neurophysiol 99:1758-1769. CrossRef Medline

Bos JH, Jongkees LB (1963) On galvanic stimulation of the labyrinth. Pract Otorhinolaryngol (Basel) 25:345-348. Medline

Branoner F, Straka H (2015) Semicircular canal-dependent developmental tuning of translational vestibulo-ocular reflexes in Xenopus laevis. Dev Neurobiol 75:1051-1067. CrossRef Medline

Clarke AH (2010) Laboratory testing of the vestibular system. Curr Opin Otolaryngol Head Neck Surg 18:425-430. CrossRef Medline
Cohen B, Martinelli GP, Ogorodnikov D, Xiang Y, Raphan T, Holstein GR, Yakushin SB (2011) Sinusoidal galvanic vestibular stimulation (sGVS) induces a vasovagal response in the rat. Exp Brain Res 210:45-55. CrossRef Medline

Courjon JH, Precht W, Sirkin DW (1987) Vestibular nerve and nuclei unit responses and eye movement responses to repetitive galvanic stimulation of the labyrinth in the rat. Exp Brain Res 66:41-48. Medline

Curthoys IS (2010) A critical review of the neurophysiological evidence underlying clinical vestibular testing using sound, vibration and galvanic stimuli. Clin Neurophysiol 121:132-144. CrossRef Medline

Curthoys IS, Macdougall HG (2012) What galvanic vestibular stimulation actually activates. Front Neurol 3:117. CrossRef Medline

Dai C, Fridman GY, Davidovics NS, Chiang B, Ahn JH, Della Santina CC (2011) Restoration of 3D vestibular sensation in rhesus monkeys using a multichannel vestibular prosthesis. Hear Res 281:74-83. CrossRef Medline

Dai C, Fridman GY, Chiang B, Rahman MA, Ahn JH, Davidovics NS, Della Santina CC (2013) Directional plasticity rapidly improves 3D vestibuloocular reflex alignment in monkeys using a multichannel vestibular prosthesis. J Assoc Res Otolaryngol 14:863-877. CrossRef Medline

Eatock RA, Songer JE (2011) Vestibular hair cells and afferents: two channels for head motion signals. Annu Rev Neurosci 34:501-534. CrossRef Medline

Ezure K, Cohen MS, Wilson VJ (1983) Response of cat semicircular canal afferents to sinusoidal polarizing currents: implications for input-output properties of second-order neurons. J Neurophysiol 49:639-648. Medline

Ferrè ER, Longo MR, Fiori F, Haggard P (2013) Vestibular modulation of spatial perception. Front Hum Neurosci 7:660. CrossRef Medline

Fitzpatrick RC, Day BL (2004) Probing the vestibular system with galvanic stimulation. J Appl Physiol 96:2301-2316. Medline

Fitzpatrick RC, Watson SR (2015) Passive motion reduces vestibular balance and perceptual responses. J Physiol 593:2389-2398. CrossRef Medline

Fridman GY, Della Santina CC (2013) Safe direct current stimulation to expand capabilities of neural prostheses. IEEE Trans Neural Syst Rehabil Eng 21:319-328. CrossRef Medline

Fritzsch B, Straka H (2014) Evolution of mechanosensory hair cells and inner ears: identifying stimuli to select altered molecular development toward new morphologies. J Comp Physiol A Neuroethol Sens Neural Behav Physiol 200:5-18. CrossRef Medline

Galvani L (1791) De viribus electicitatis in motu musculari commentarius. Translated by Foley, M.G. as Luigi Galvani: Commentary of the Effects of Electricity on Muscular Motion. 1953, Burndy Library: Norwalk.

Goldberg JM (2000) Afferent diversity and the organization of central vestibular pathways. Exp Brain Res 130:277-297. CrossRef Medline

Goldberg JM, Smith CE, Fernández C (1984) Relation between discharge regularity and responses to externally applied galvanic currents in vestibular nerve afferents of the squirrel monkey. J Neurophysiol 51:1236-1256. Medline

Gong W, Merfeld DM (2000) Prototype neural semicircular canal prosthesis using patterned electrical stimulation. Ann Biomed Eng 28:572-581. CrossRef Medline

GrafW, Simpson JI (1981) The relations between the semicircular canals, the optic axis, and the extraocular muscles in lateral-eyed and frontal-eyed animals. In: Progress in oculomotor research, developments in neuroscience, Vol 12 (Fuchs A, Becker W, eds), pp 411-420. Elsevier: New York.

Grasso C, Orsini P, Bruschini L, Manzoni D, Barresi M (2013) A new technique to investigate vestibulo-spinal reflexes. Arch Ital Biol 151:54-66. CrossRef Medline

Highstein SM, Goldberg JM, Moschovakis AK, Fernández C (1987) Inputs from regularly and irregularly discharging vestibular nerve afferents to secondary neurons in the vestibular nuclei of the squirrel monkey. II. Correlation with output pathways of secondary neurons. J Neurophysiol 58:719-738. Medline

Hitzig E (1871) Über galvanischen Schwindel. Arch Ohren-, Nasen- Kehlkopfheilk 5/6:34-37.

Holler S, Straka H (2001) Plane-specific brainstem commissural inhibition in frog second order semicircular canal neurons. Exp Brain Res 137: 190-196. CrossRef Medline

Honrubia V, Hoffman LF, Sitko S, Schwartz IR (1989) Anatomic and physiological correlates in bullfrog vestibular nerve. J Neurophysiol 61:688701. Medline

Hsu LJ, Zelenin PV, Orlovsky GN, Deliagina TG (2012) Effects of galvanic vestibular stimulation on postural limb reflexes and neurons of spinal postural network. J Neurophysiol 108:300-313. CrossRef Medline

Kaufmann AK, Gensberger K, Dietrich H, Branoner F, Banchi R, Chagnaud 
BP, Straka H (2013) Mechanistic basis and spatiotemporal specificity of galvanic vestibular stimulation in Xenopus laevis. Soc Neurosci Abstr 39:164.04.

Kim J (2013a) Head movements suggest canal and otolith projections are activated during galvanic vestibular stimulation. Neuroscience 253: 416-425. CrossRef Medline

Kim J (2013b) Tonic eye movements induced by bilateral and unilateral galvanic vestibular stimulation (GVS) in guinea pigs. Brain Res Bull 90: 72-78. CrossRef Medline

Kim J, Curthoys IS (2004) Responses of primary vestibular neurons to galvanic vestibular stimulation (GVS) in the anaesthetised guinea pig. Brain Res Bull 64:265-271. CrossRef Medline

Kim KS, Minor LB, Della Santina CC, Lasker DM (2011) Variation in response dynamics of regular and irregular vestibular-nerve afferents during sinusoidal head rotations and currents in the chinchilla. Exp Brain Res 210:643-649. CrossRef Medline

Lambert FM, Beck JC, Baker R, Straka H (2008) Semicircular canal size determines the developmental onset of angular vestibuloocular reflexes in larval Xenopus. J Neurosci 28:8086-8095. CrossRef Medline

Lambert FM, Malinvaud D, Gratacap M, Straka H, Vidal PP (2013) Restricted neural plasticity in vestibulospinal pathways after unilateral labyrinthectomy as the origin for scoliotic deformations. J Neurosci 33: 6845-6856. CrossRef Medline

LeRoy C (1755) Où l'on rend compte de quelques tentatives que l'on a faites pour guérir plusieurs maladies par l'électricité. Hist Acad Roy Sciences (Paris), Mémoire Math Phys 60:87-95.

Lewis RF (2015) Advances in the diagnosis and treatment of vestibular disorders: psychophysics and prosthetics. J Neurosci 35:5089-5096. CrossRef Medline

Minor LB, Goldberg JM (1991) Vestibular nerve inputs to the vestibuloocular reflex: A functional ablation study in the squirrel monkey. J Neurosci 11:1636-1648. Medline

Mitchell DE, Dai C, Rahman MA, Ahn JH, Della Santina CC, Cullen KE (2013) Head movements evoked in alert rhesus monkey by vestibular prosthesis stimulation: implications for postural and gaze stabilization. PLoS One 8:e78767. CrossRef Medline

Nieuwkoop PD, Faber J (1994) Normal table of Xenopus laevis (Daudin): a systematical and chronological survey of the development from the fertilized egg till the end of metamorphosis. New York: Garland.

Phillips JO, Ling L, Nie K, Jameyson E, Phillips CM, Nowack AL, Golub JS, Rubinstein JT (2015) Vestibular implantation and longitudinal electrical stimulation of the semicircular canal afferents in human subjects. J Neurophysiol 113:3866-3892. CrossRef Medline
Precht W (1978) Neuronal operations in the vestibular system: studies of brain function. Berlin Heidelberg: Springer.

Purkinje J (1820) Beiträge zur näheren Kenntnis des Schwindels. Med Jahrb k u k Staates (Wien) 6:23-35.

Ramlochansingh C, Branoner F, Chagnaud BP, Straka H (2014) Tricaine methanesulfonate (MS-222) as an effective anesthetic agent for blocking sensory-motor responses in Xenopus laevis tadpoles. PLoS ONE 9:e101606. CrossRef Medline

Schneider E, Glasauer S, Dieterich M (2002) Comparison of human ocular torsion patterns during natural and galvanic vestibular stimulation. J Neurophysiol 87:2064-2073. Medline

Shanidze N, Lim K, Dye J, King WM (2012) Galvanic stimulation of the vestibular periphery in guinea pigs during passive whole body rotation and self-generated head movement. J Neurophysiol 107:2260-2270. CrossRef Medline

St George RJ, Fitzpatrick RC (2011) The sense of self-motion, orientation and balance explored by vestibular stimulation. J Physiol 589:807-813. CrossRef Medline

Straka H, Dieringer N (2000) Convergence pattern of uncrossed excitatory and inhibitory semicircular canal-specific inputs onto second-order vestibular neurons of frogs. Exp Brain Res 135:462-473. CrossRef Medline

Straka H, Dieringer N (2004) Basic organization principles of the VOR: lessons from frogs. Prog Neurobiol 73:259-309. CrossRef Medline

Straka H, Simmers J (2012) Xenopus laevis: an ideal experimental model for studying the developmental dynamics of neural network assembly and sensory-motor computations. Dev Neurobiol 72:649-663. CrossRef Medline

Straka H, Baker R, Gilland E (2001) Rhombomeric organization of vestibular pathways in larval frogs. J Comp Neurol 437:42-55. CrossRef Medline

Straka H, Baker R, Gilland E (2002a) The frog as a unique vertebrate model for studying the rhombomeric organization of functionally identified hindbrain neurons. Brain Res Bull 57:301-305. CrossRef Medline

Straka H, Holler S, Goto F (2002b) Patterns of canal and otolith afferent input convergence in frog second-order vestibular neurons. J Neurophysiol 88:2287-2301. CrossRef Medline

Straka H, Vibert N, Vidal PP, Moore LE, Dutia MB (2005) Intrinsic membrane properties of vertebrate vestibular neurons: Function, development and plasticity. Prog Neurobiol 76:349-392. CrossRef Medline

Straka H, Fritzsch B, Glover JC (2014) Connecting ears to eye muscles: evolution of a 'simple' reflex arc. Brain Behav Evol 83:162-175. CrossRef Medline

Thompson DM, Koppes AN, Hardy JG, Schmidt CE (2014) Electrical stimuli in the central nervous system microenvironment. Annu Rev Biomed Eng 16:397-430. CrossRef Medline

Wardman DL, Fitzpatrick RC (2002) What does galvanic vestibular stimulation stimulate? Adv Exp Med Biol 508:119-128. CrossRef Medline 